\title{
Turbulent boundary layer to single-stream shear layer: the transition region
}

\author{
By SCOTT C. MORRIS $\dagger$ AND JOHN F. FOSS \\ Department of Mechanical Engineering, Michigan State University, East Lansing, MI 48823, USA
}

(Received 7 February 2003 and in revised form 16 June 2003)

This communication presents the results and conclusions of an experimental study of the near-separation region of a single-stream shear layer. The momentum thickness at separation $(x=0)$ was $\theta_{0}=9.6 \mathrm{~mm}$, with Reynolds number $R e_{\theta}=4650$. Boundary layer separation was caused by a sharp $90^{\circ}$ edge. Detailed single- and multipoint measurements of the velocity field were acquired at the streamwise locations $0<x / \theta_{0}<100$. This represents the transition region between two of the canonical turbulent shear flows: the zero-pressure-gradient turbulent boundary layer and the single-stream shear layer. From the viewpoint of a separating boundary layer, the results describe how the turbulent flow reacts to a sudden change in wall boundary conditions. From the viewpoint of the developed shear layer, the results describe the transition to the self-similar region. The data acquired suggest that the initial shear layer instability occurs in the region very near separation $\left(x \approx \theta_{0}\right)$, and that it involves only the vorticity filaments which originate in the near-wall region of the upstream boundary layer. This 'near-wall region' roughly defines the origin of a narrow wedge-shaped domain that was identified from the velocity statistics. This domain is termed the 'sub-shear layer'. The statistics of the velocity field in the region bounded by the sub-shear layer and the free-stream flow were found to represent the normative continuation of the upstream boundary layer. The sub-shear layer has been found to exhibit many of the standard features observed in fully developed shear layers. For example, velocity measurements on the entrainment side of the shear layer indicate that large-scale motions with spanwise coherence were observed. The streamwise dependence of the dominant frequency, convection velocity, and spanwise velocity correlation have been documented in order to characterize the sub-shear layer phenomenon.

\section{Introduction}

A single-stream shear layer is of interest as one of the canonical turbulent shear flows and it is also important in technological applications. The region near the separation edge of the shear layer can serve as the archetype flow for a backwardfacing step, a jet exhausting from a plane surface, and other flows characterized by the interaction between a separating primary flow and the induced secondary or entrained flow. This communication presents the results of an experimental investigation of the near-separation region with a turbulent boundary layer at separation. A schematic

$\dagger$ Author to whom correspondence should be addressed; Present address: Department of Aerospace and Mechanical Engineering, University of Notre Dame, Notre Dame, IN 46556, USA; morris.65@nd.edu 


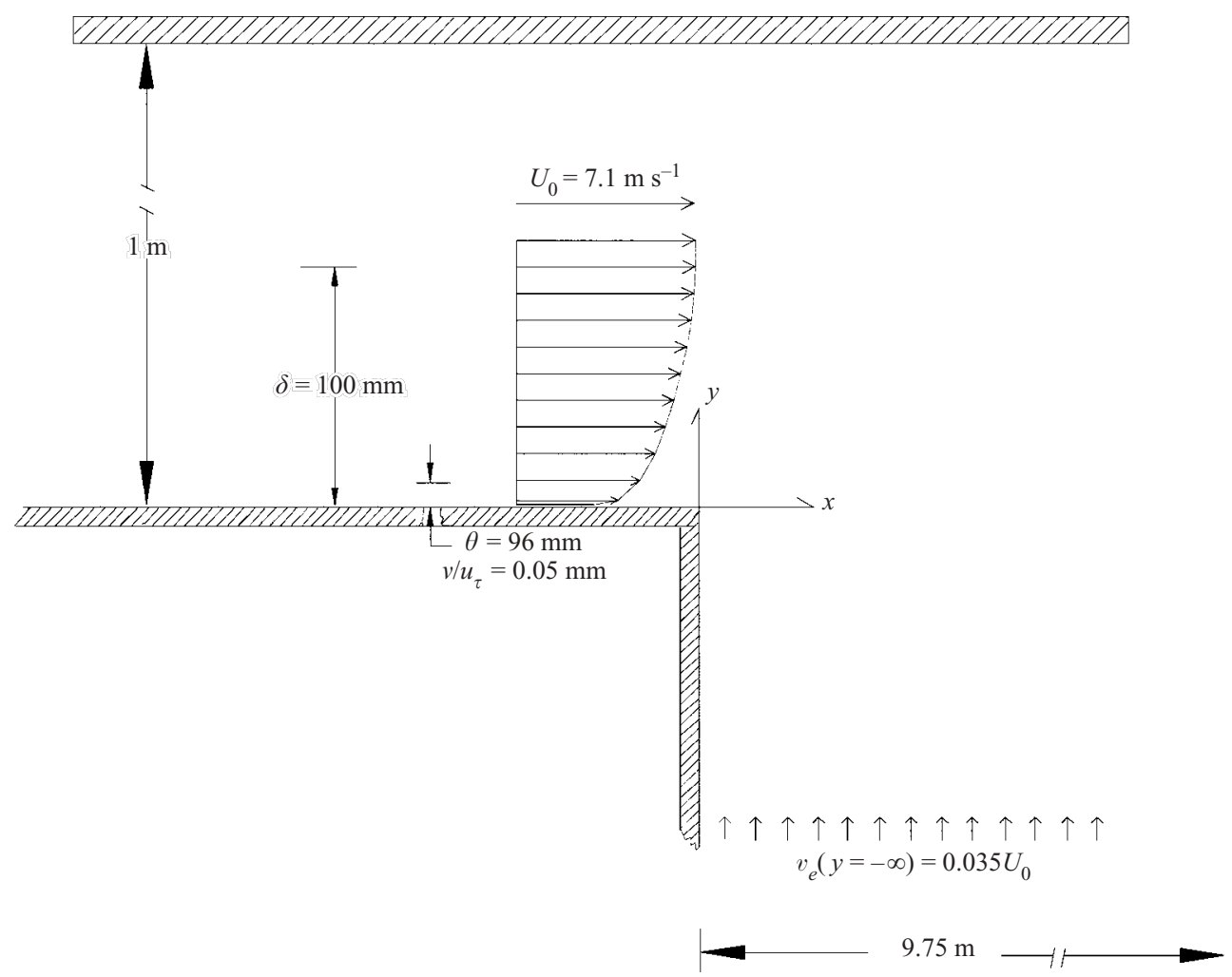

FIGURE 1. Schematic representation of near-separation region.

representation of the area of interest and the adopted coordinate system is shown in figure 1. Measurements were acquired in the region $0<x / \theta_{0}<100$, where $\theta_{0}$ represents the momentum thickness of the boundary layer at $x=0$. This study is distinctive with respect to the existing literature because of the relatively high Reynolds number (based on $\left.\theta_{0}\right)$ of the separating boundary layer $(R e=4650)$, and because the large $\theta_{0}$ value $(9.6 \mathrm{~mm})$ permitted detailed measurements to be acquired in the region very near separation.

The domain of interest can be viewed as the transition region between two wellstudied canonical flow fields: a flat-plate turbulent boundary layer and a single-stream shear layer. This leads to two possible perspectives from which to view the acquired data. The first perspective is to view the region shown in figure 1 as a boundary layer with a sudden change in the wall boundary conditions. The data then serve to answer the question: how does a turbulent boundary layer react to this abrupt 'removal' of the wall boundary condition? A related question was addressed using a moving-wall geometry in Hamelin \& Alving (1996). It will be demonstrated in the following sections of this paper (in agreement with the results of Hamelin \& Alving) that the 'outer region' of the separated boundary layer remains statistically identical to the upstream (canonical) boundary layer for several integral length scales in the streamwise direction. The insensitivity of the outer region of the boundary layer to the sudden 'removal' of the bounding wall is note-worthy, and this insensitivity is expected to be of interest to researchers who study boundary layers as well as separated flows. 
In the second viewpoint the near-separation region is considered to be the developing region of the shear layer. The data then answer the question: how does a self-similar single-stream shear layer develop from its parent boundary layer? Implicit in the concept of self-similarity is the idea that the initial conditions are 'forgotten' (Townsend 1975). The near-separation region has been extensively studied for the case of laminar boundary layers at separation. For example, Ho \& Huerre (1984) provide a thorough review and a substantial list of both single-stream and two-stream shear layer references. Many of these studies examine the near-separation region using linear stability theory. The results of the present work will be compared and contrasted to this established theory.

There have been a number of investigations which have looked at shear layers with various inflow conditions. See, for example, Bradshaw (1966), Foss (1977), Hussain \& Zedan (1978), Slessor, Bond \& Dimotakis (1998), Browand \& Latigo (1979), and Dziomba \& Fiedler (1985). These studies were conducted with laminar and turbulent (tripped) separating boundary layers with Reynolds number $\left(R e_{\theta}\right)$ less than 1000 . These references report experiments in which the upstream boundary condition is modified and the 'effect' of this modification on the developed downstream flow is documented. A common characteristic of these studies is that the near-separation region was not described in detail.

In summary, the present research effort serves to fill a gap in the present understanding regarding the physical mechanisms which take place when a singlestream shear layer originates from a relatively high-Reynolds-number boundary layer. The experimental programme was divided into four parts. The first was a characterization of the boundary layer at the separation point $(x=0)$. These experiments utilized single- and multiple-sensor hot-wire probes to document many of the single-point statistics of the velocity and vorticity fields. These data serve to document the inflow boundary conditions to the control volume of interest. The results of the second group of experiments document the single-point statistics of the velocity in the region $0<x / \theta_{0}<100$. In the third group of experiments, single- and multi-point measurements of velocity were used to document many of the features of the unsteady irrotational flow on the low-speed entrained side of the shear layer. In the fourth part, a rake of eight hot-wire sensors was used to document the spanwise $(z)$ correlations of the velocity field at various $(x, y)$ locations. The results of the four groups of experiments will be described following an overview of the experimental methods and the single-stream shear layer facility. Note that the results from the experiments will be presented with limited discussion. The paper ends with a list of conclusions and with a more detailed discussion of the results. This format of presentation was chosen so that the discussion could be provided from the perspective of incorporating all of the experimental data.

\section{Experimental methods and apparatus}

\subsection{Single-stream shear layer facility}

A large single-stream shear layer facility at the Turbulent Shear Flows Laboratory at Michigan State University was used for the present experiments. A schematic representation is shown in figure 2 . Note that the height 'out of the page' of the entire flow system was $1.94 \mathrm{~m}$.

A constant-speed blower (not shown) moves air from the laboratory to the point labelled Primary inflow. The air then passes through the flow conditioners. These consist of a $2 \mathrm{~m}$ wide honeycomb ( $3 \mathrm{~mm}$ cell $\times 25 \mathrm{~mm}$ length) section and three 


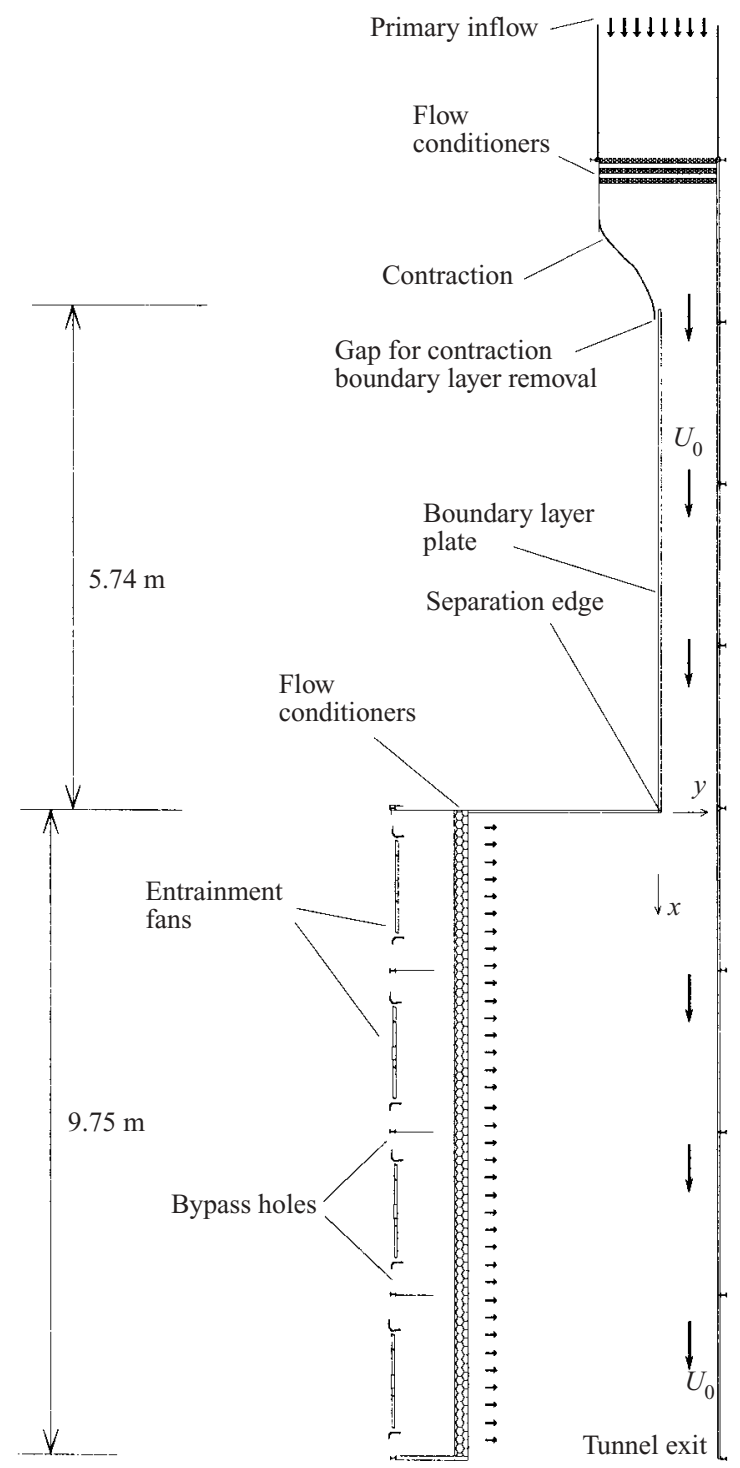

FIGURE 2. Schematic of the single-stream shear layer facility. Note that view is rotated $90^{\circ}$ with respect to figure 1 .

turbulence control screens with 8 wires $/ \mathrm{cm}$. A contraction is located $1.2 \mathrm{~m}$ downstream of the screens. This reduces the width of the flow area to $1.1 \mathrm{~m}$. The boundary layer plate begins at the end of the contraction. A small gap $(0.12 \mathrm{~m})$ between the end of the contraction and the leading edge of the boundary layer plate allows the removal of the contraction boundary layer. This point of the tunnel and all downstream locations are at nearly zero gauge pressure with respect to the laboratory ambient. The primary flow moves through the $0.98 \mathrm{~m}$ wide boundary layer development section. The boundary layer plate is $5.74 \mathrm{~m}$ long; the free-stream velocity was $7.1 \mathrm{~m} \mathrm{~s}^{-1}$. The boundary layer plate was laminated with $1 \mathrm{~mm}$ thick PVC sheeting to provide a smooth boundary condition. The boundary layer plate terminated at the separation point which was created using a machined $90^{\circ}$ edge. The free-stream velocity was 
measured during all experiments by monitoring the differential pressure drop across the planar contraction. This pressure difference was acquired during all experiments and it was found to vary by less than $0.5 \%$ over the duration of the experimental programme.

The shear layer test section begins at the separation edge. The primary flow moves through the $9.75 \mathrm{~m}$ test section length with constant velocity $\left(U_{0}\right)$. An entrainment flow stream was directed perpendicular to the primary flow stream on the low-speed side of the shear layer. This flow was created using four $1.2 \mathrm{~m}$ diameter axial propeller-type fans with an AC speed controller. This fan type is designed to move large volumes of air at relatively low pressure rise $(\sim 25 \mathrm{~Pa})$. For this reason, bypass holes were created such that the fans would operate at design conditions to prevent unsteadiness from blade stall. That is, most of the air moved by the fans is recirculated back to the laboratory. The 'plenum' between the fans and the flow conditioning is maintained at a pressure which is slightly higher than the atmospheric value. The entrainment flow conditioning is identical to the primary flow conditioning. The lateral location of the entrainment screen closest to the shear layer is $y=-2.75 \mathrm{~m}$, using the coordinate system shown with origin at the separation edge.

The entrainment fans were adjusted to provide the correct entrainment rate for a shear layer at zero pressure gradient. This was verified using hot-wire sensors in the primary flow at several streamwise locations. That is, the fans were adjusted such that $\partial U_{0} / \partial x \approx 0$. The resulting entrainment velocity was found to be $v_{e}=0.035 U_{0} \approx$ $0.25 \mathrm{~m} \mathrm{~s}^{-1}$.

The exit of the tunnel was open to the laboratory. The nearest obstruction to the outflow was a building wall located $6.1 \mathrm{~m}$ from the tunnel exit. The ceiling and floor of the laboratory were respectively located approximately $2 \mathrm{~m}$ above and below the shear layer test section. These conditions provided sufficient space to turn the exiting flow without disturbing the shear layer at the end of the test section.

\subsection{Instrumentation}

Time series of the fluid velocity were measured using single- and multi-sensor hot-wire probes. The sensor elements were made from $5 \mu \mathrm{m}$ diameter tungsten wire. The total wire length was $3 \mathrm{~mm}$, with a $1 \mathrm{~mm}$ region on each side that was plated with $50 \mu \mathrm{m}$ of copper to support the $1 \mathrm{~mm}$ active portion of the wire. The sensors were controlled using DISA 55M anemometers with a heating ratio of 1.7. The frequency response was always greater than $20 \mathrm{kHz}$. All sensors were calibrated before (pre) and after (post) each experiment with a maximum of three hours between calibrations. Unless the pre and post calibrations agreed to $2 \%$ or better the data were retaken.

Measurements acquired in the separating boundary layer also include twocomponent $(u, v)$ data acquired using an X-wire probe. Additionally, vorticity time series data were acquired using a compact four-wire probe. A full description of these probes and processing algorithms can be found in Foss \& Haw (1990), Wallace \& Foss (1995), and Morris (2002). A thorough review of hot-wire techniques is given by Bruun (1985).

\section{Experiment 1: characterization of the boundary layer}

The study of the near-separation region began with detailed measurements that were acquired very near to the separation point $(x \approx 0)$. These data and their interpretation serve to document the inflow boundary conditions to the separated flow discussed in the following sections. The investigation of the boundary layer was divided into 
two experiments. First, measurements to identify possible effects of separation on the boundary layer upstream of the separation edge were executed. Second, point statistics in the boundary layer were measured with enough detail to ensure that a nearly canonical zero-pressure-gradient boundary layer existed and that it compared well with standard measurements as reported in the literature.

\subsection{Shear layer to boundary layer 'communication'}

The purpose of this experiment was to answer the question: do the pressure fluctuations from the shear layer significantly alter the characteristics of the boundary layer at separation?. In other words, do the large-scale spanwise coherent motions in the shear layer cause a measurable change in the boundary layer characteristics upstream of separation? The answer to this question for the condition of a laminar boundary layer at separation is discussed in Holmes, Lumley \& Berkooz (1992). Their results suggest that the shear layer does influence the separating boundary layer for laminar separation. In contrast, it will be argued from the present data that this is not true for the present (high-Reynolds-number turbulent) boundary layer.

It is known (see e.g. Browand \& Trout 1980) that the velocity field in the irrotational fluid near a planar shear layer is unsteady. This is a result of the large-scale motions which exist within the turbulent region. If these motions also affect the boundary layer flow before separation, it is intuitively reasonable that a non-zero correlation between velocities in the boundary layer and in the entrainment stream would provide evidence that the shear layer was affecting the boundary layer. It is noted, however, that statistical quantities such as linear correlations do not indicate causation, and should only be used as an indicator that a mechanistic coupling exists between the two regions.

Simultaneous hot-wire measurements were taken in both the entrainment stream and the boundary layer flows to test this hypothesis. A single hot-wire sensor was traversed in the entrainment stream near the separation point with the sensor axis aligned with the $z$-direction such that the components of velocity in the $(x, y)$ plane would be recovered. Note that the $w$ component should be quite small in the essentially two-dimensional entrainment region. The entrainment probe was traversed through a range of locations in the region $0<x / \theta_{0}<20, y<0$. An X-wire probe was simultaneously placed in the boundary layer in the region $0<y / \theta_{0}<10,-4<x / \theta_{0}<0$, such that the $u$ and $v$ components of velocity were recovered. Time series data from the X-wire and the entrainment probe were recorded for 40 seconds at $5000 \mathrm{~Hz}$. The standard normalized cross-correlation as a function of $\tau_{n}$, the discrete time delay variable, is defined as

$$
R\left(\tau_{n}\right)=\frac{\overline{v_{1}\left(t_{n}\right) v_{2}\left(t_{n}+\tau_{n}\right)}}{\tilde{v}_{1} \tilde{v}_{2}} .
$$

The variable $v_{1}$ is the $u$ or $v$ component of velocity measured from the $\mathrm{X}$-wire, $v_{2}$ is one of the signals from the entrainment probes, and the overbar represents the time average. The tilde represents the RMS value of that signal.

The results of these calculations have shown nearly zero correlation values for all of the above measurement locations. That is, there was no measurable correlation between the two signals for any of the spatial locations measured. A second calculation was performed in which a low-pass filter was used to allow only frequencies less than, for example, $f_{c}=100 \mathrm{~Hz}$ (where $f_{c} \theta(0) / U_{0}=0.135$ ) to be correlated. This type of filtered cross-correlation has been shown to be effective in detecting nonzero correlations in highly turbulent flow fields which contain broad-band spectral 


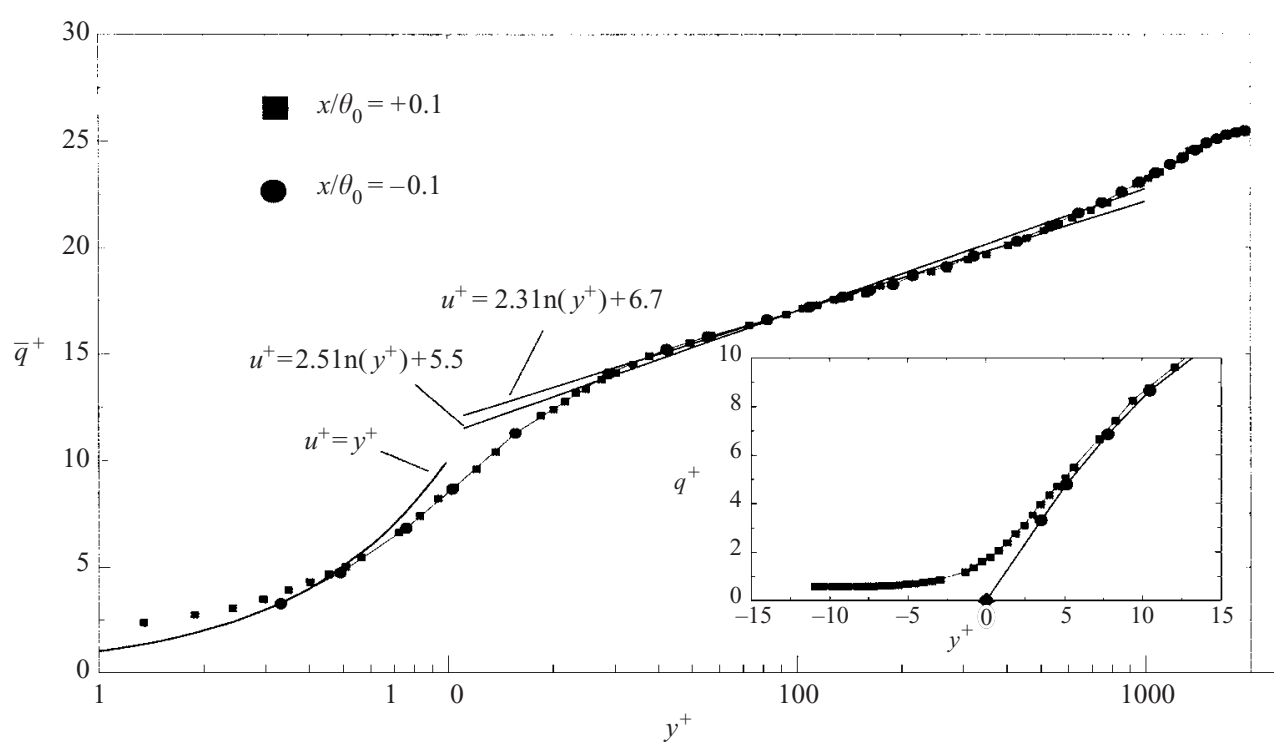

FIGURE 3. Boundary layer mean velocity. The $y^{+}=0$ point $(+)$ on the inset figure was added for clarity, based upon the no-slip condition. Note that $U_{0} / u_{\tau}=25.5$.

information; see e.g. Naguib (1992). These cross-correlations, however, were also found to be negligible in magnitude for all of the acquired data.

It is inferred from the above observations that the upstream 'communication' is not measurable in a separating turbulent boundary layer. It is therefore reasonable to assume that the measurable characteristics of a high-Reynolds-number turbulent boundary layer upstream from a fixed separation point with zero pressure gradient are identical to those which would exist if the separation were not present. These conclusions are further supported by the results of the following section, which presents detailed velocity measurements for $x \approx 0$. As indicated earlier, this result is in contrast to observations described in Holmes et al. (1992) for a separating laminar boundary layer. Further discussion will be given in the conclusions section.

\subsection{Boundary layer statistics near $x=0$}

The single-point statistics of the boundary layer in the region very near separation were measured using a single hot-wire sensor. The probe was calibrated and traversed through the boundary layer at the streamwise locations $x / \theta_{0}=-0.1$ and +0.1 . Each data point was acquired at a sampling frequency of $5000 \mathrm{~Hz}$ for $t=30 \mathrm{~s}$, where $t U_{0} / \theta_{0}=2.2 \times 10^{4}$. The sensor was aligned in the $z$-direction, such that the magnitude of the velocity in the $(x, y)$-plane was recovered:

$$
q(t)=\sqrt{u^{2}+v^{2}}
$$

Most authors do not make the distinction between $u$ and $q$ in single-probe hot-wire measurements. This is because the $v$ component is typically small compared to $u$ in wall-bounded flows. The emphasis on $q$ in the present work is important because the surveyed region $(x>0, y<0)$, will be dominated by the $v$ component of velocity. In contrast, for the region $(x \approx 0, y>0)$ the approximation $q \approx u$ is nearly exact.

The time average and standard deviation of the velocity measurements will be denoted by $\bar{q}$ and $\tilde{q}$ respectively. The profiles $\bar{q}\left(x= \pm 0.1 \theta_{0}, y\right)$ are shown in figure 3 . Note that these data are normalized by the viscous units $\left(u_{\tau}=\sqrt{\tau_{w} / \rho}\right.$ for velocity and 


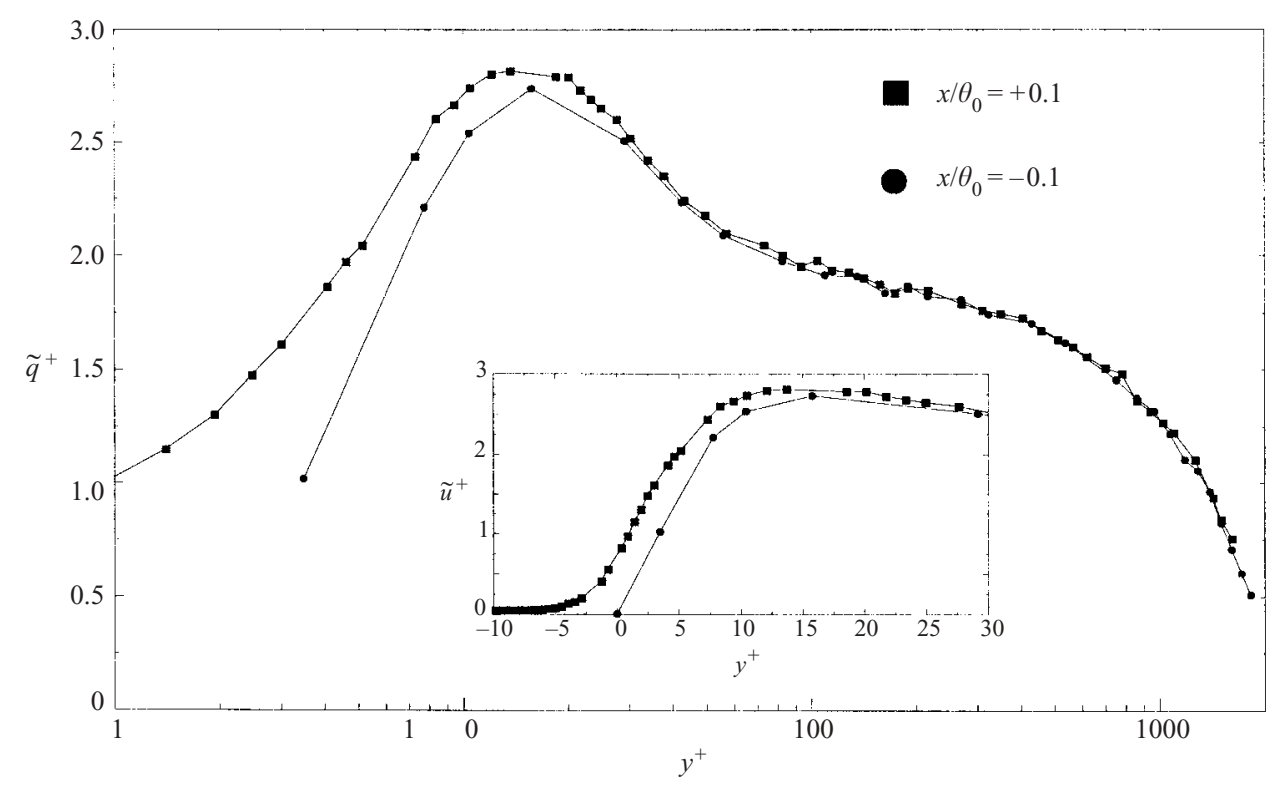

FIGURE 4. Boundary layer velocity RMS.

$v / u_{\tau}$ for $y$, where $\tau_{w}$ is the time-averaged wall shear stress, $\rho$ density and $v$ viscosity) in $\log$-linear coordinates. The wall shear stress was computed from the slope of a linear fit of the data points closest to the wall at $x / \theta_{0}=-0.1$. Note that the closest location to the wall is $y^{+} \approx 3.2$, or roughly $y \approx 150 \mu \mathrm{m}$, which is far enough from the wall to ignore proximity effects in the hot-wire readings. A typical log-linear region can be observed through the range $40<y^{+}<800$. This region roughly approximates the standard fit $q^{+}=2.5 \ln \left(y^{+}\right)+5.5$. The present data are best represented by $q^{+}=2.3 \ln \left(y^{+}\right)+6.7$ for $30<y^{+}<500$.

It is of interest to compare the two velocity profiles which have been acquired. The measured $x / \theta_{0}= \pm 0.1$ mean values agree for all locations where $y^{+}>5(y>0.25 \mathrm{~mm})$. The inset to the figure shows the deviation of the data from the two traverses for $y^{+}<5$ in linear coordinates. The deviation between the two profiles indicates a strong streamwise gradient in velocity given that the $x$ displacement of the sensor for the two traverses was $0.19 \theta_{0}(1.8 \mathrm{~mm})$. For example, $\Delta \bar{q} / \Delta x \approx 400\left(\mathrm{~s}^{-1}\right)$ at $y=0$.

The integral statistics of interest, which were calculated from the mean profile, were the momentum thickness $\theta=9.62 \mathrm{~mm}$, and the displacement thickness $\delta^{*}=12.6 \mathrm{~mm}$. The Reynolds number based on momentum thickness was $R e_{\theta}=4650$. Additional parameters are the friction coefficient and the shape factor, which are defined as $C_{f}=2\left(u_{\tau} / U_{0}\right)^{2}$ and $H=\delta^{*} / \theta$, respectively. These were determined to be $C_{f}=0.00295$ and $H=1.31$.

The $\tilde{q}\left(x= \pm 0.1 \theta_{0}, y\right)$ profiles in viscous units are shown in figure 4. It is observed that the majority of the $y^{+}>0$ region is invariant in the $\tilde{q}$ data, as observed in figure 3 . Specifically, $\tilde{q}$ is invariant for $y^{+}>30$, whereas $\bar{q}$ is invariant for $y^{+}>5$. Note that the maximum value for the $x / \theta=-0.1$ data is $\tilde{q} / u_{\tau}=2.74$. Other researchers (for example Klewicki \& Falco 1990) have found the peak in the fluctuation intensity to be $\sim 2.9$ for similar Reynolds numbers. This discrepancy can be explained by the relatively large sensor length used in the present study. Specifically, the hot-wire length in viscous units was $\lambda^{+}=20 v / u_{\tau}$, whereas Klewicki \& Falco (1990) recommend 


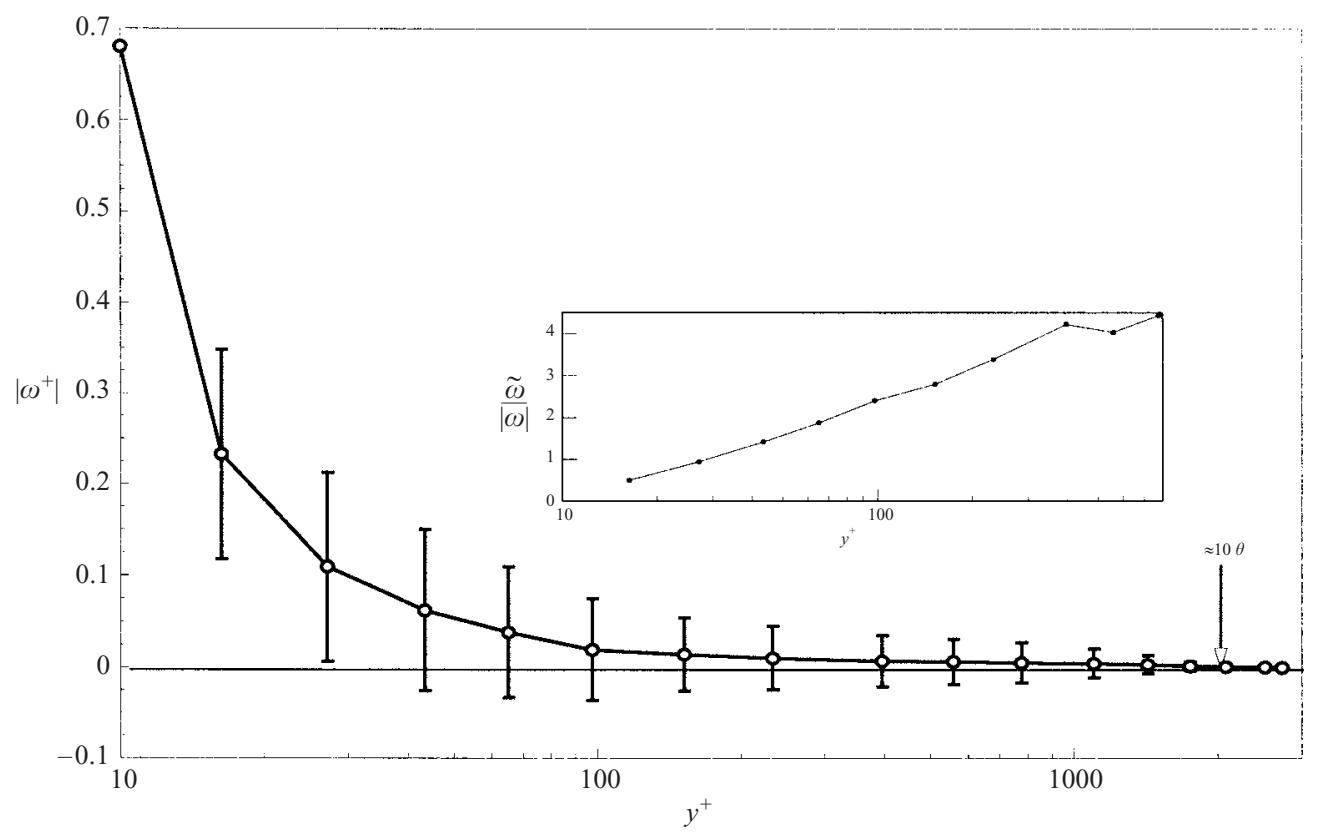

FiguRE 5. Mean and RMS of vorticity at separation. -o-, mean value from slope of single sensor traverse. Error bars denote \pm 1 standard deviation of vorticity calculated from the four-wire vorticity probe time series.

a sensor length of less than $10 v / u_{\tau}$ in order to avoid spatial averaging of the velocity fluctuations.

The spanwise vorticity in the boundary layer is also a variable of interest. The magnitude of the time-averaged vorticity in viscous units is approximated by $\left|\overline{\omega_{z}^{+}}\right|=\partial \bar{q}^{+} / \partial y^{+}$. The slope of the mean velocity profile shown in figure 3 was computed; see figure 5. The standard deviations of the fluctuations in spanwise vorticity, $\tilde{\omega}_{z}$, were measured using the four-wire vorticity probe; these data are also shown on figure 5 . Note that the spatial resolution of the probe is established by the distance between the two straight wires. This is nominally $1 \mathrm{~mm}$ ( 20 viscous wall units in the present study). The measured values of $\tilde{\omega}_{z}$ are consistent with the data presented in Klewicki, Falco \& Foss (1992) and references therein. These data show that both positive and negative values of vorticity were realized for $y^{+}>20$. This can be compared with the near-wall region wherein only one sign of vorticity that is consistent with that of the mean shear is observed. The vorticity fluctuations normalized by the local mean value: $\tilde{\omega}_{z} /\left|\overline{\omega_{z}}\right|$ have been plotted for the region $10<y^{+}<800$ as the inset to figure 5. Note that the values of $\tilde{\omega}_{z} /\left|\overline{\omega_{z}}\right|$ are uncertain for $y^{+}>800$ because both the numerator and denominator are approaching zero. The data show that the relative fluctuation levels of vorticity increase approximately exponentially (linear in semi-log coordinates) throughout the 'log region' $\left(10<y^{+}<600\right)$ of the boundary layer. The comparison of the two representations of $\tilde{\omega}_{z}$ in figure 5 are instructive in showing that the absolute fluctuation levels of vorticity decrease with increasing $y^{+}$, yet the values are increasing with respect to the local mean vorticity.

Qualitative aspects of the vorticity filament topology can be inferred from these measurements and the solenoidal condition of the vorticity vector field. At a small distance from the wall the filaments are necessarily perpendicular to the local shear 


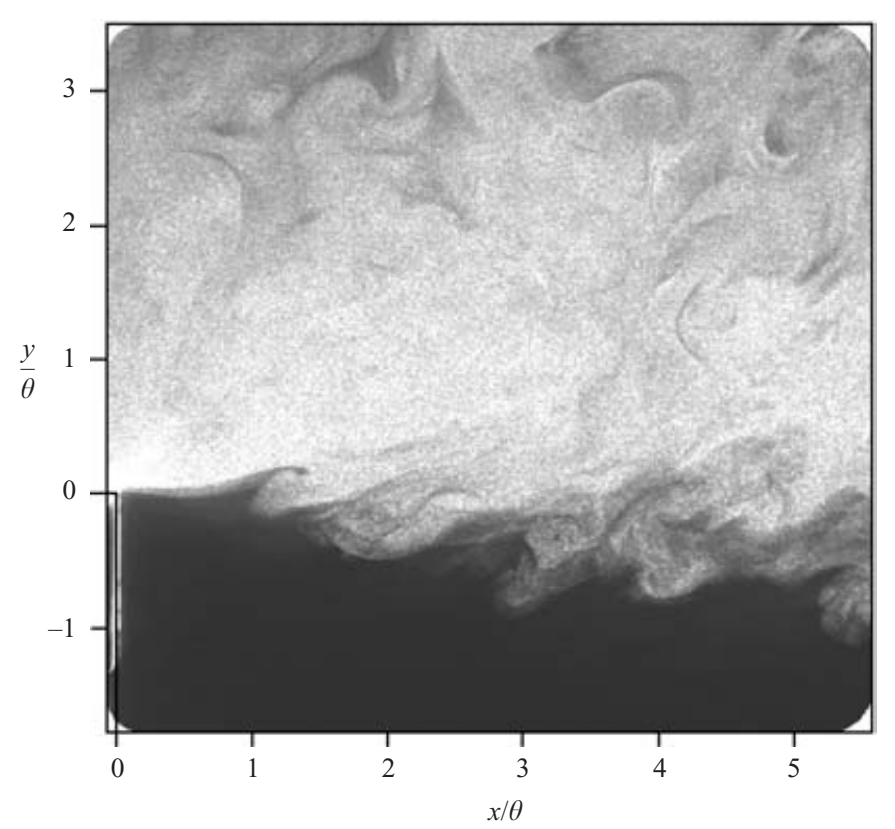

FIGURE 6. Sample flow visualization of the near-separation region.

stress vector, and parallel to the surface. The observation that $\omega_{z}(t)<0$ in the near-wall region implies that the near-wall filament topology is relatively simple. In contrast, the measurements farther from the wall $\left(y^{+} \sim 20\right)$ indicate that the vorticity filaments must be sufficiently complex or 'twisted' such that vorticity values of both sign are realized at a given $y$ location. Farther from the wall, the statistics of the velocity and vorticity fields exhibit many of the properties of isotropic turbulence; see, for example, Saddoughi \& Veeravalli (1994) for examples of velocity statistics which support local isotropy in the log region of boundary layers. These features of the vorticity field will be considered in the following sections which describe the near-separation region of the boundary layer.

\section{Experiment 2: point statistics and the 'sub-shear layer'}

A flow visualization image of the near-separation region is shown in figure 6 . This digital image was acquired using a Kodak $1 \mathrm{k} \times 1 \mathrm{k}$ camera and a nominally $1 \mathrm{~mm}$ thick laser light sheet oriented in the $(x, y)$-plane. The boundary layer flow was seeded using an oil-fog system located upstream of the boundary layer leading edge. The unmarked entrainment flow provided the contrast between the two fluid streams. Specifically, the marked primary fluid appears white, and the clean entrainment fluid appears dark in the figure. Note the scale and orientation with respect to figure 1. Specifically, recall that the boundary layer thickness $\delta$ is of order $10 \theta$. This picture is not repeatable in detail because the flow is turbulent in this region. However, the general features represented and described are common to all realizations.

Several interesting observations can be made from this visualization. First, a streakline from the separation point can be easily identified. This shows a 'wavy' or 'flag-flapping' type of behaviour. The first disturbance to the streakline occurs at a streamwise location of $x \approx \theta_{0}$. The 'size' of this motion appears to be of order $0.5 \theta_{0}$. This observation is consistent with the 'communication' results described previously. 


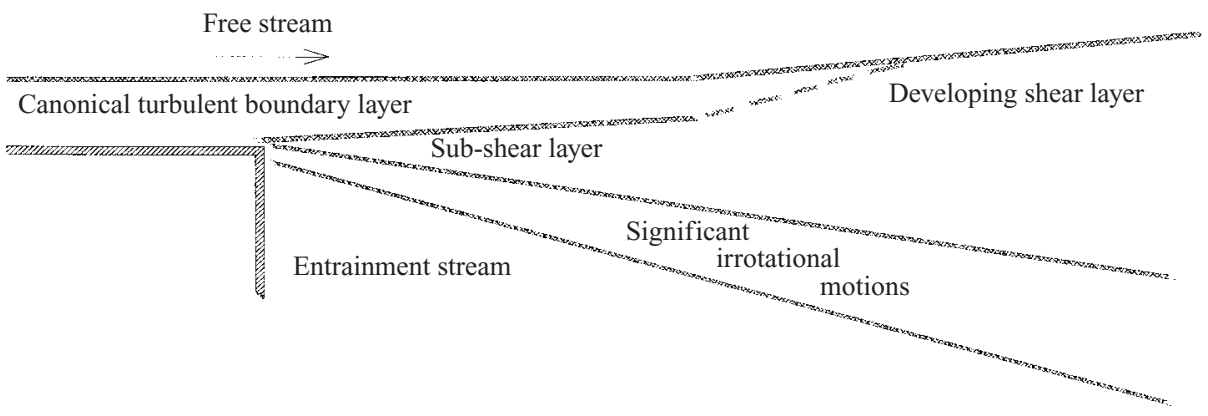

FIGURE 7. Diagram of flow regions; not to scale.

That is, it would seem unlikely that the pressure fluctuations from this first disturbance or the downstream disturbances would influence the upstream boundary layer given their relatively small scale. Note also that the asymmetry of the shear layer is evident from the movement of the marked fluid into the entrainment region $(y<0)$, whereas very little clean fluid is observed for $y>0$. The visually apparent 'growth' of the shear layer is clearly at a length scale which is significantly smaller than the boundary layer thickness.

Based on these observations, and equipped with the data to be presented in this and subsequent sections, several distinct regions of the flow field have been identified. These are shown schematically in figure 7 . These regions and their defining characteristics will provide a conceptual framework and vocabulary which will be useful in describing the results to follow. The canonical turbulent boundary layer is the region which exhibits stochastic values of the velocity field that reflect the evolution of a boundary layer that is still attached to a flat plate. The sub-shear layer represents the turbulent region where the flow is measurably different from that if the bounding wall were present. The region marked significant irrotational fluctuations represents the domain where measurements of the velocity fluctuations exhibit essentially no high-frequency (small-scale) motions. It is inferred that the unsteady aspects of these motions are driven only by the unsteady pressure field created by the shear layer. Lastly, the transition between the sub-shear layer and the developing shear layer are not well defined. Hence no such boundaries are drawn in figure 7.

Point statistics were acquired using a single hot-wire probe which was traversed across the shear layer at 12 streamwise locations. The data were acquired at $5 \mathrm{kHz}$ for $60 \mathrm{~s}$. If the characteristic velocity is taken as $U_{0} / 2$, and if the shear layer width is taken to be $6 \theta(x)$, then the 60 s represents 500 to 2000 shear layer widths for the 12 locations. The sensor was aligned in the $z$-direction to recover the time series of $q$ as described in the previous section. Note that in the high-speed free stream the sensor responds to $q=u=U_{0}$, and in the entrainment stream the sensor responds to $q=v=v_{e}$. The time-average velocity $(\bar{q})$ and the standard deviation of the fluctuations $(\tilde{q})$ were then computed from the time series values. The mean velocity is shown in figure 8 as an ' $x, y$ plot', and in figure 9 as a contour plot. The values of $\tilde{q}$ are shown in figures 10 and 11 in $x, y$ and contour formats, respectively. Note that the boundary of the sub-shear layer region, identified qualitatively in figure 7 , can be roughly described by the $\tilde{q} / U_{0}=0.087$ contour in figure 11 .

A striking feature observed in these data is the nearly streamwise-invariant boundary layer statistics. That is, the gradients $\partial() / \partial x$ that are known to be small in boundary layers are also very small downstream of separation. As a specific 


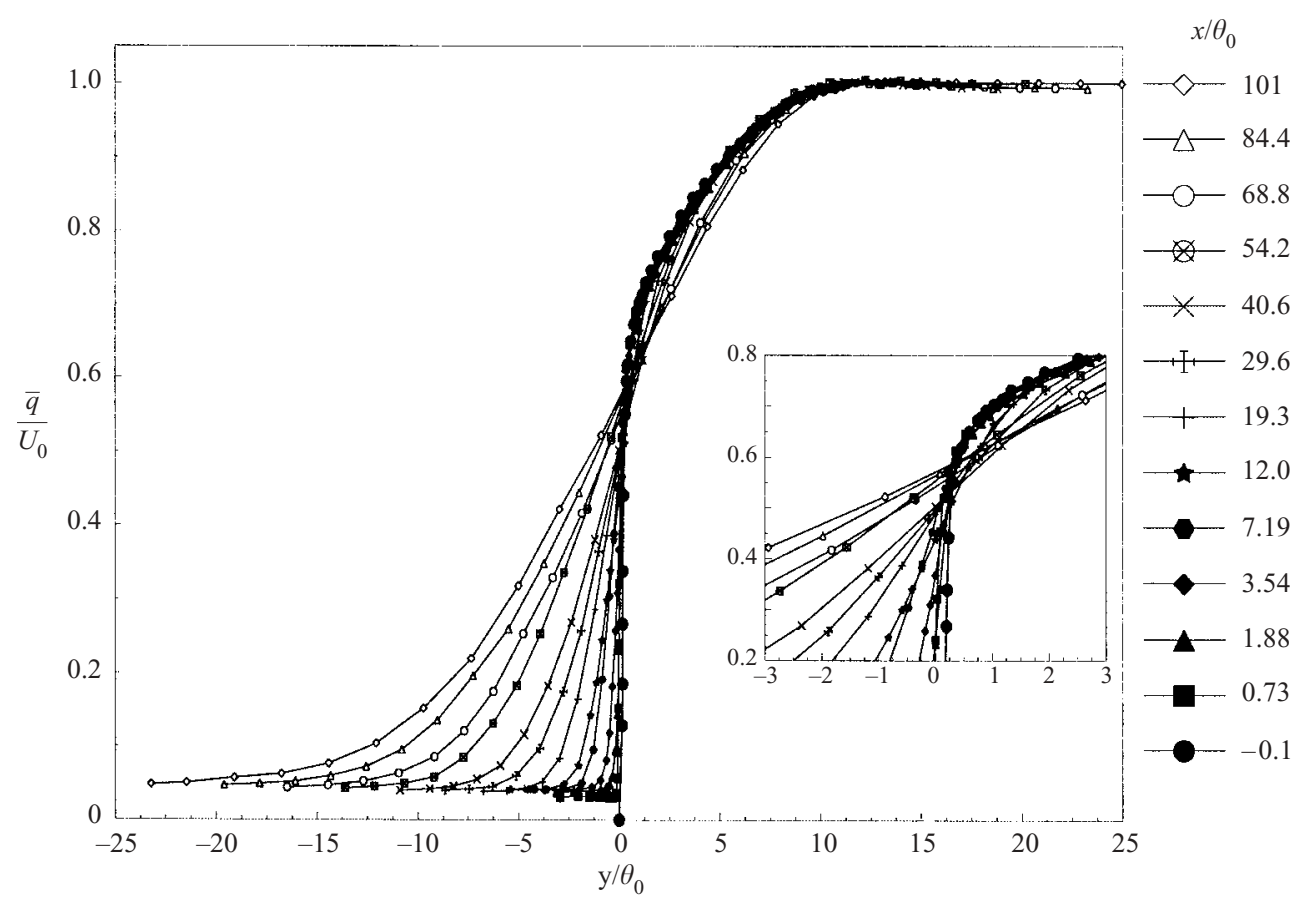

FIGURE 8 . Mean velocity profiles for $0<x / \theta_{0}<100$.

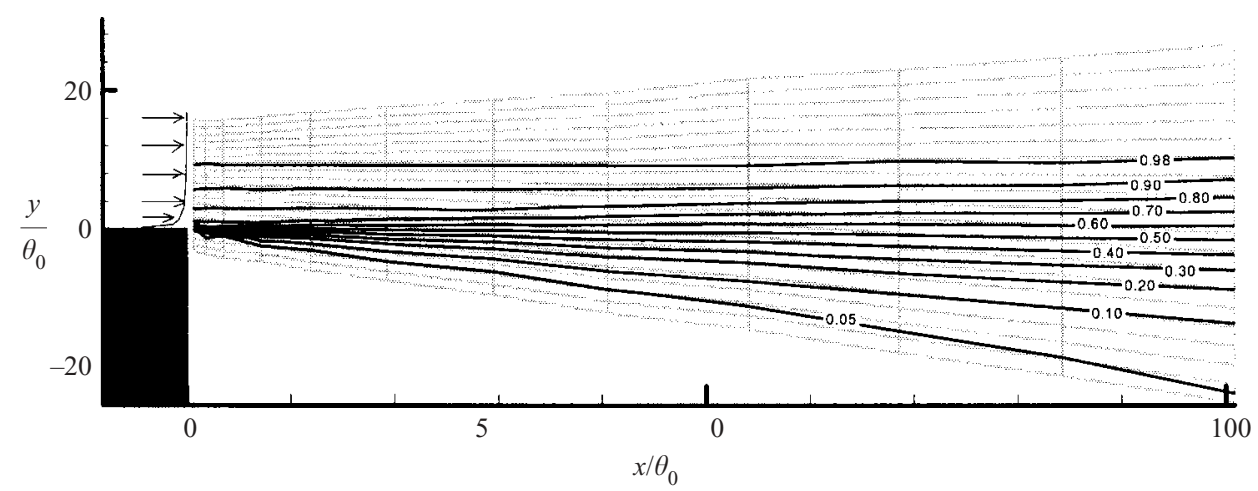

Figure 9. Contour plot of mean velocity, $\bar{q} / U_{0}$. The grey 'grid' represents the $(x, y)$ locations where data were collected.

example, the mean profiles are identical to the boundary layer profile for $y / \theta_{0}>2$ in the range $0<x / \theta_{0}<29$. The profiles of $\tilde{q}$ are streamwise invariant for $y / \theta_{0}>4$ in the range $0<x / \theta_{0}<29$. These features are shown by the nearly streamwise-invariant contours in figures 9 and 11, or by close examination of the individual profiles in figures 8 and 10. Several histograms of velocity are given in figure 12 for the points: $\left(x / \theta_{0}, y / \theta_{0}\right)=(0.73,2),(7.2,2)$ and $(19,2)$. Although differences can be noted, the similarity of these histograms provides a specific example of the nominal streamwise invariance of the moments of the velocity field.

It is noteworthy that these results are consistent with those of Hamelin \& Alving (1996). In that reference, a moving belt was used to remove the inner layer of an $R e_{\theta}=2800$ turbulent boundary layer in order to study the stochastic evolution of 


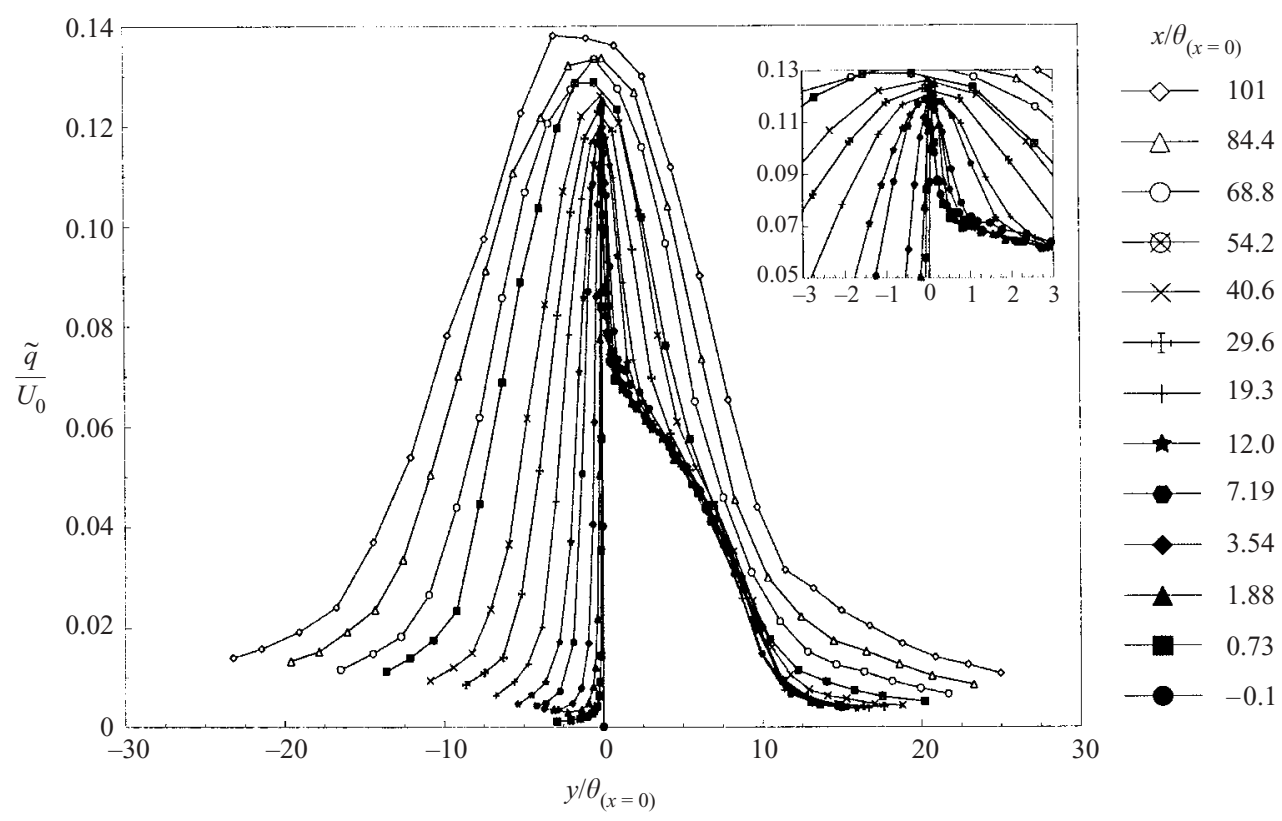

FIGURE 10. RMS of velocity for $0<x / \theta_{0}<100$.

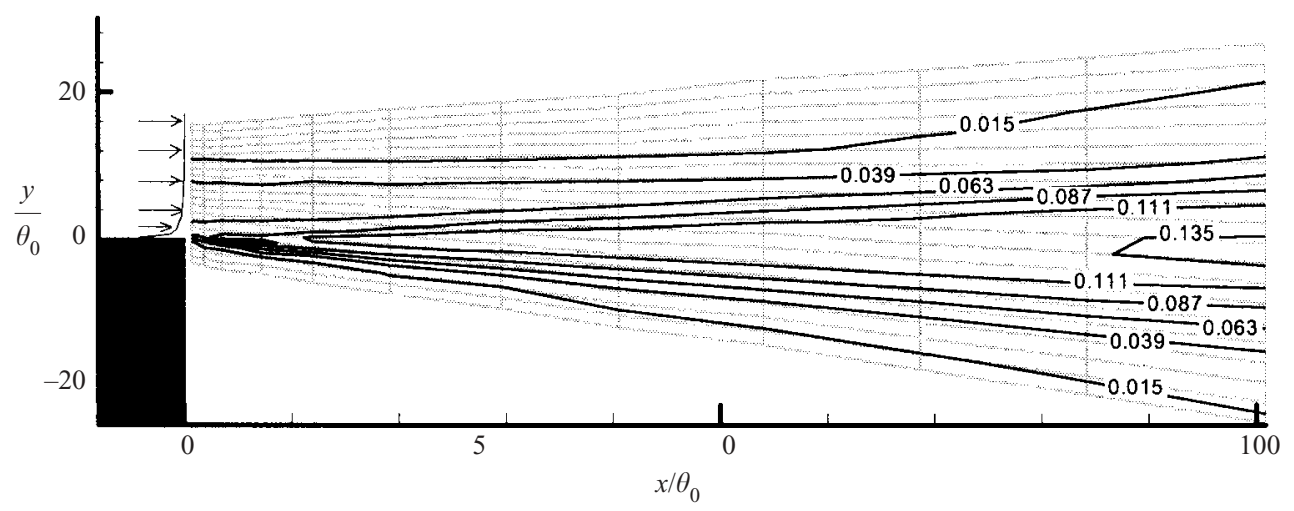

FIGURE 11. Contour plot of velocity RMS $\tilde{q} / U_{0}$.

the outer scales. The present work is similar in that the streamwise evolution of the boundary layer is measured after an abrupt change in wall boundary conditions. In the case of the moving-wall boundary condition, the mean flow field was streamwise invariant for $y / \theta_{0}>2$ in the range $0<x / \theta_{0}<34$ (see figure 5 of Hamelin \& Alving). The RMS of the velocity fluctuations at a given $y$ location was streamwise invariant for $y / \theta_{0}>4$ in the range $0<x / \theta_{0}<30$ (figure 7 of Hamelin \& Alving). These regions of streamwise invariance are nearly identical to those realized in the present experiment.

Two variables of interest were calculated from the mean velocity profiles: the momentum thickness of the shear layer $\theta(x)$ and the maximum slope of the mean velocity. The calculation of $\theta$ requires the integration of the streamwise component of velocity across the shear layer. Because the magnitude of the velocity was measured as opposed to strictly the $u$ component (see equation (2)), an arbitrary cut-off of $\bar{q} / U_{0}=0.05$ was used to end the integration on the low-speed side. This is a common 


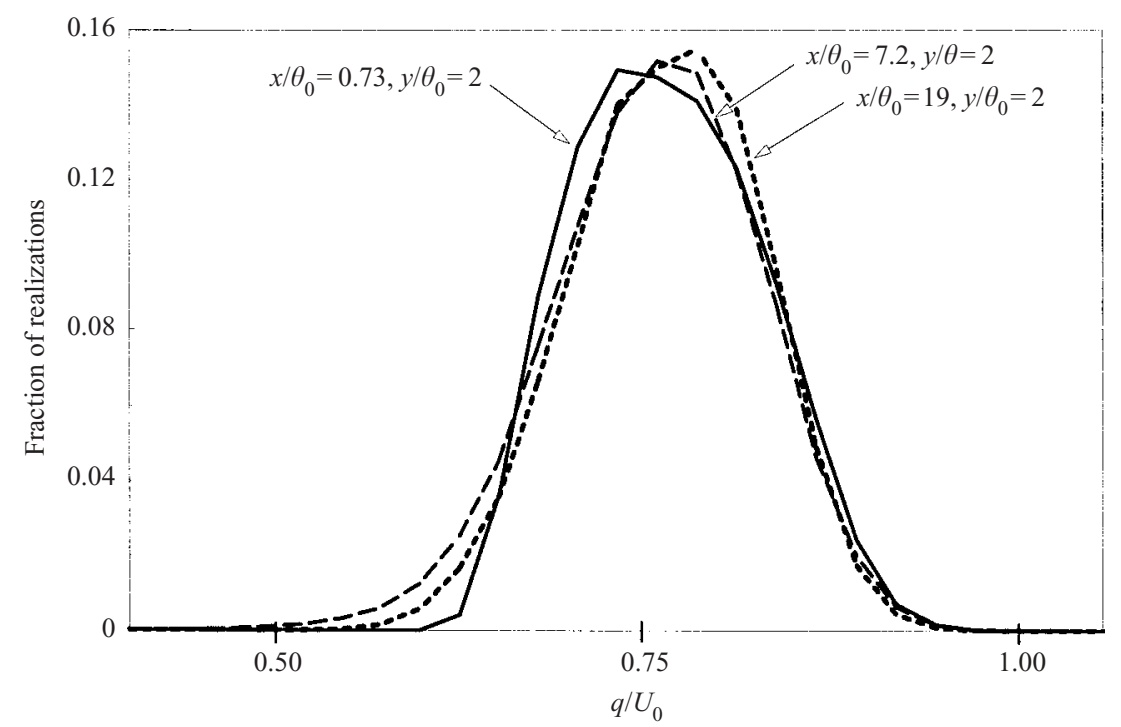

FIGURE 12. Streamwise evolution of velocity histogram at constant $y / \theta_{0}=2.0$.

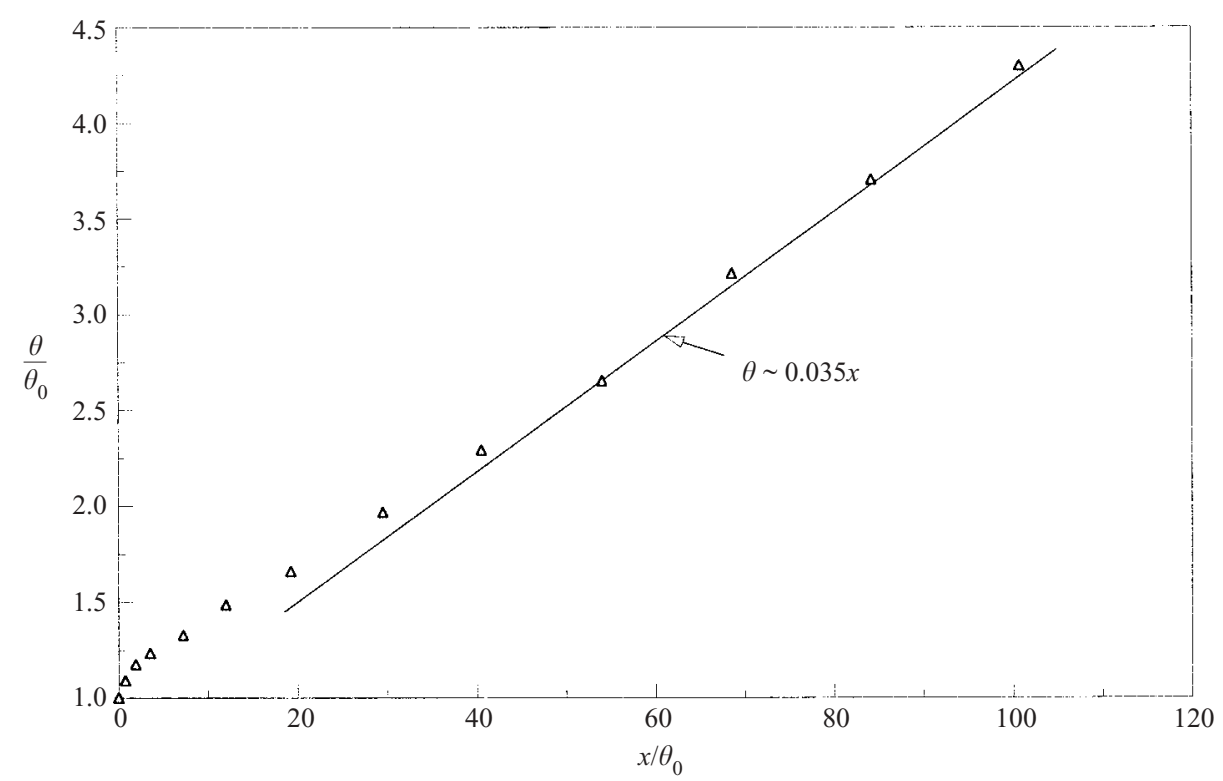

FIGURE 13. Momentum thickness vs. $x / \theta_{0}$.

technique used by, for example, Ali et al. (1985) and Hussain \& Zaman (1985). These results are shown in figure 13. The growth rate in the region $x / \theta_{0}>60$ was found to be 0.035 as measured previously by Foss (1994). Note that Hussain \& Zaman (1985) found a linear growth rate of 0.032 . The second variable derived from the point statistics that will be of interest in subsequent sections is the maximum slope of the velocity profile. These data are shown in $\log -\log$ format in figure 14 for streamwise locations $\left(1.8<x / \theta_{0}<550\right)$. This extended domain shows that the 


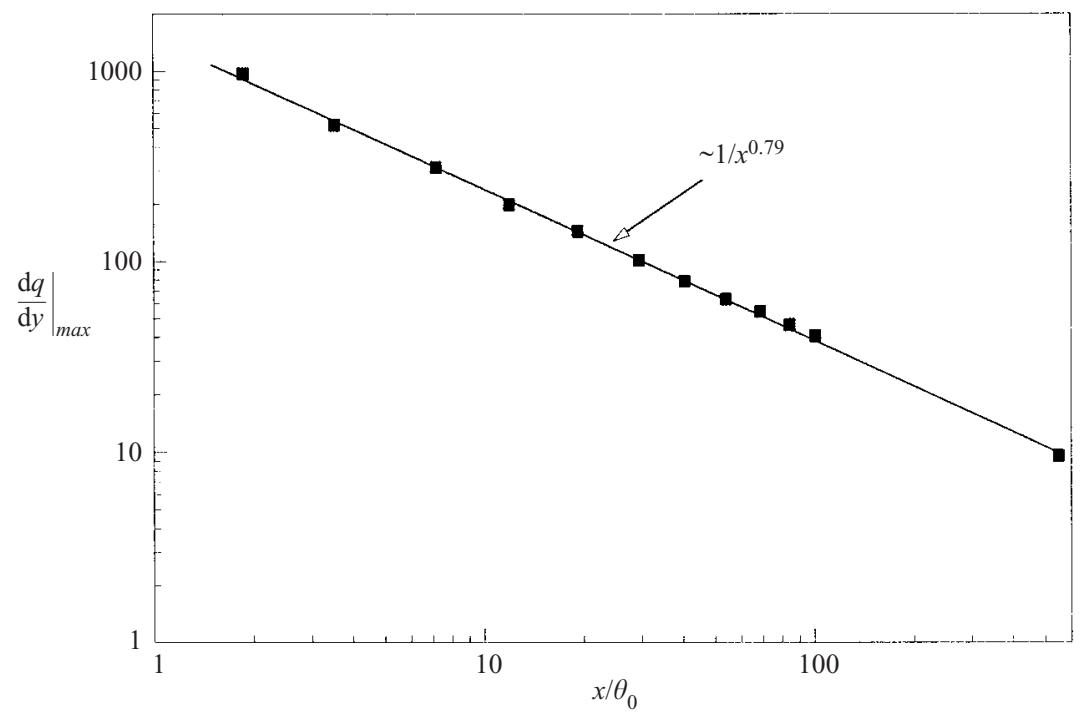

FIgURE 14. Maximum velocity gradient vs. $x / \theta_{0}$.

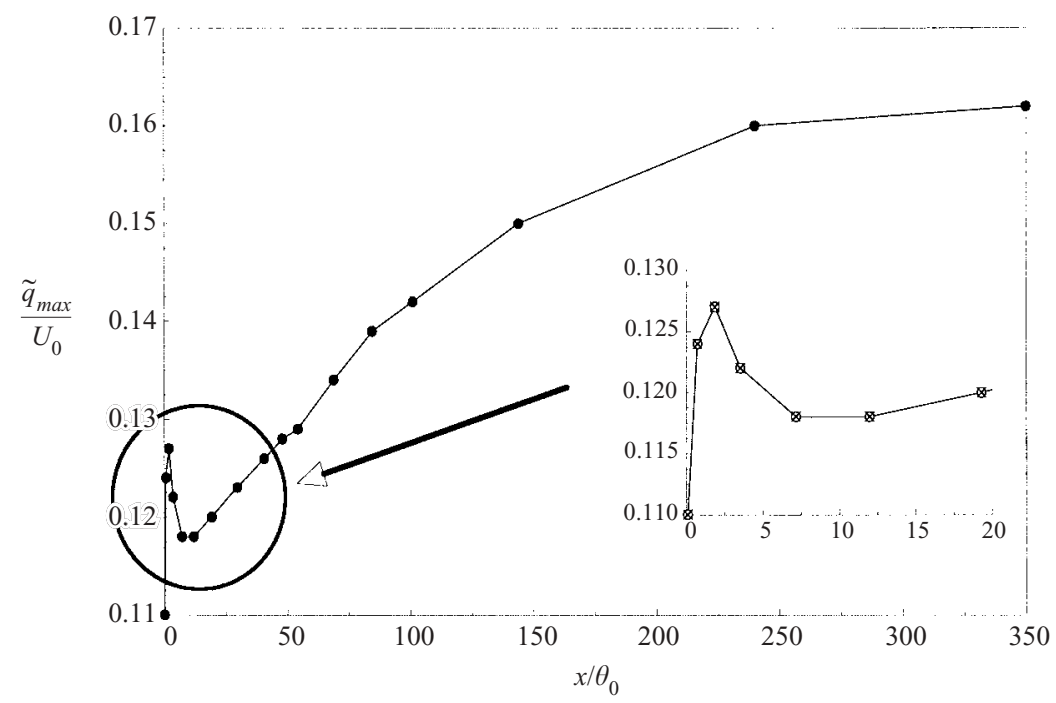

FIGURE 15. Peak value of velocity RMS as a function of streamwise location.

function $(\mathrm{d} q / \mathrm{d} y)_{\max } \sim 1 / x^{0.79}$ agrees reasonably well throughout the sub-shear layer region as well as farther downstream.

A variable of interest is the maximum value of $\tilde{q}$ obtained at a given streamwise location. This information was obtained from the data shown in figure 10, and plotted in figure 15. The most interesting feature of these data is that the local maximum value is realized at $x / \theta_{0} \approx 2$. This peak occurs at a lateral position of $y / \theta_{0}=0.15$. Previously, the existence of a local maximum has only been observed in shear layers with a laminar separating boundary layer, whereas the tripped or high-Reynoldsnumber boundary layers lead to a monotonic increase in $\tilde{q}$; see Foss (1977) and Hussain \& Zedan (1978). The present data support the concept that the sub-shear 
layer region very near the separation point is a viscously dominated flow region which shares many of the characteristics of low-Reynolds-number separations. It is inferred that this detailed feature of the flow field near the separation edge was previously not observed given the small dimensions of $\theta_{0}$ in those experiments.

As a final note regarding the sub-shear layer, the observations made also provide insight into the transition length required in order to obtain a self-similar shear layer. Specifically, it is known that shear layers which originate from a laminar boundary layer require a streamwise development of $1000 \theta_{0}$ in order to be self-similar (Bradshaw 1966). In contrast, self-similarity is observed in shear layers which originate from a turbulent boundary layer at streamwise locations less than $150 \theta_{0}$, as observed in the present experiments, as well as in the literature (see e.g. Hussain \& Zaman 1985). Dimotakis \& Brown (1976) suggested that the streamwise distance to self-similarity is related to the wavelength of the initial stability $\left(\lambda_{0}\right)$, and not to the initial momentum thickness $\left(\theta_{0}\right)$. They also indicate that the case of turbulent separation will probably have shorter length scales compared to $\theta_{0}$. This can be expressed as the ratio $\lambda_{0} / \theta_{0}$. This value has been found to be $\lambda_{0} / \theta_{0} \approx 10$ for laminar separation, and $\lambda_{0} / \theta_{0} \approx 1$ in the present study (see figure 6). The present result clearly supports the inference made by Dimotakis \& Brown. Further discussion will be given in the conclusions section.

\section{Experiment 3: shear layer diagnostics from entrainment region measurements}

A feature of the single-stream shear layer is the ubiquitous large-scale coherent motions. These motions are characterized by a correlation length in the spanwise (homogeneous) direction that is many times larger than the local shear layer thickness. The existence, physical makeup, and dynamic significance of these large scales are still topics of debate and research. The origin of these motions is believed to be the inflectional instability of the shear layer velocity profile. The flow visualization and point statistics described in the previous section indicated that this instability does not influence the entire separating boundary layer. Rather, it is suggested that the influence of these motions is limited to the region identified as the sub-shear layer. A simple method of detecting these large-scale motions is to acquire velocity time series in the irrotational flow field near the shear layer. Many studies have made use of the fact that the time signature of velocity in both the high-speed and low-speed streams can be used to identify features of the large-scale motions within the shear layer; see for example, Browand \& Weidman (1976). Koochesfahani et al. (1979), Browand \& Trout (1985), and Narayanan \& Hussain (1996). The efficacy of this technique is based upon the pressure fluctuations, which are mechanistically linked to the large-scale motions. This leads to easily detectable fluctuations in the velocity field of the irrotational flow which bounds the vortical shear layer.

The observations made in the entrainment stream will be described in three subsections: the streamwise dependence of the convection velocity of the coherent motions, the spectral properties of the entrainment stream, and the frequency and scaling of the initial instability.

\subsection{Convection speed of the coherent motions}

A variable of interest is the convection velocity $U_{c}$ of the spanwise motions. Many investigators of both single- and two-stream shear layers have found the convection velocity to be the mean velocity of the two streams with equal density. See for example, Dimotakis (1986) and references therein. The ratio would be predicted to be $U_{c} / U_{0}=0.5$ in the single-stream shear layer. The time-averaged convection velocity 


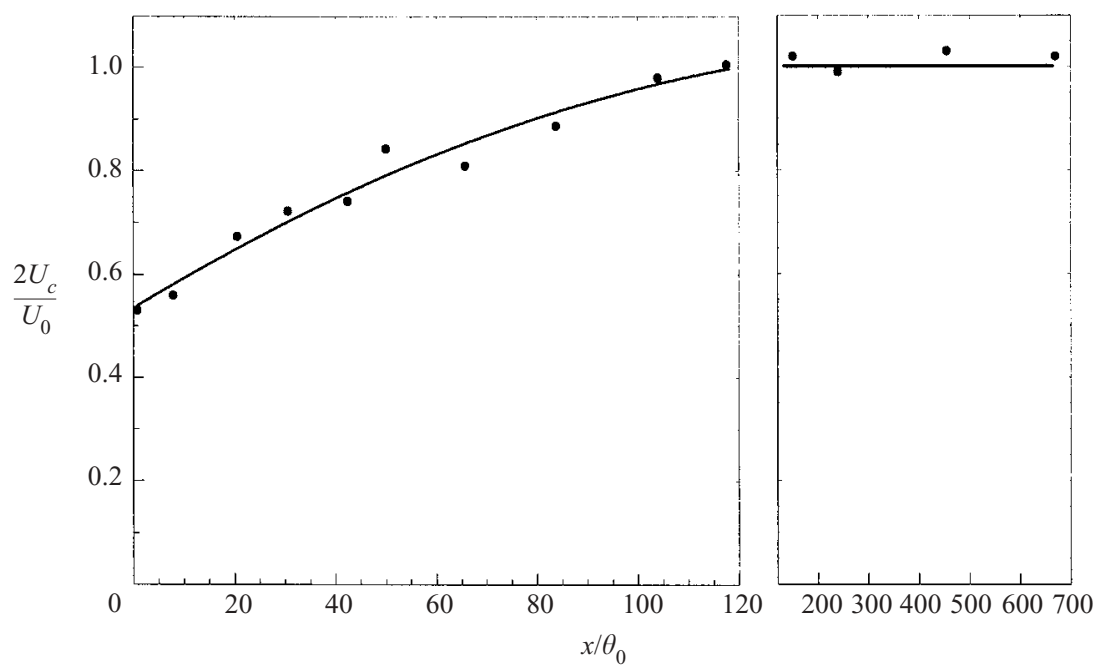

Figure 16. Convection velocity of spanwise motions. The break in the $x$-axis is used to show the $x / \theta_{0}<120$ region in more detail.

was measured from time series data of two hot wires placed in the entrainment stream with a streamwise displacement $\Delta x$. The lateral locations where $\tilde{q}(y) \approx 0.015 U_{0}$ were determined to have significant signal amplitude in the irrotational region. The timedelayed cross-correlation of the two hot-wire signals was computed from these time series to locate the time delay to the first observed positive peak: $\Delta t$. The average convection velocity is defined as $U_{c}=\Delta x / \Delta t$.

Figure 16 shows the normalized convection velocity $2 U_{c}(x) / U_{0}$. Of interest is the low value of 0.54 near $x=0$ and the increase to the expected value of 1.0 for $x / \theta_{0}>120$. It is inferred from this result that only the vorticity from the near-wall region participates in an inflectional instability in the region very near separation, and that the percentage of the vorticity (on average) that participates in the shear layer instability increases in the $x$-direction. The former inference is clearly supported by the independent visualization image of figure 6 . Additional statements can be made to support this inference. The first observation is that the velocity gradients are very steep in the region $x=y=0$ which lead to length scales which are considerably smaller than the local integral scales. Since the length scale of the most amplified motions will scale with the velocity gradient at the point of inflection, it is reasonable that only the fluid in the $y \approx 0$ region will participate in the instability.

A second argument to support this assertion is related to the vorticity field of the turbulent boundary layer upstream of separation. It is known that the wall confines the movement of the vorticity filaments in the near-wall region, and as a result they are relatively organized compared to the outer portion of the boundary layer (see Klewicki et al. 1992). Note also the large values of $\tilde{\omega}_{z} /\left|\overline{\omega_{z}}\right|$ in the outer region in figure 5. It can be inferred from these observations that the 'organized' near-wall vorticity participates in the first instability, whereas the 'disorganized' outer motions do not.

It is possible to calculate how much of the vorticity participates (on average) in the first instability based on the above observations. Given the relatively small contribution of the $y$ component of velocity to the vorticity in the near-wall region, 


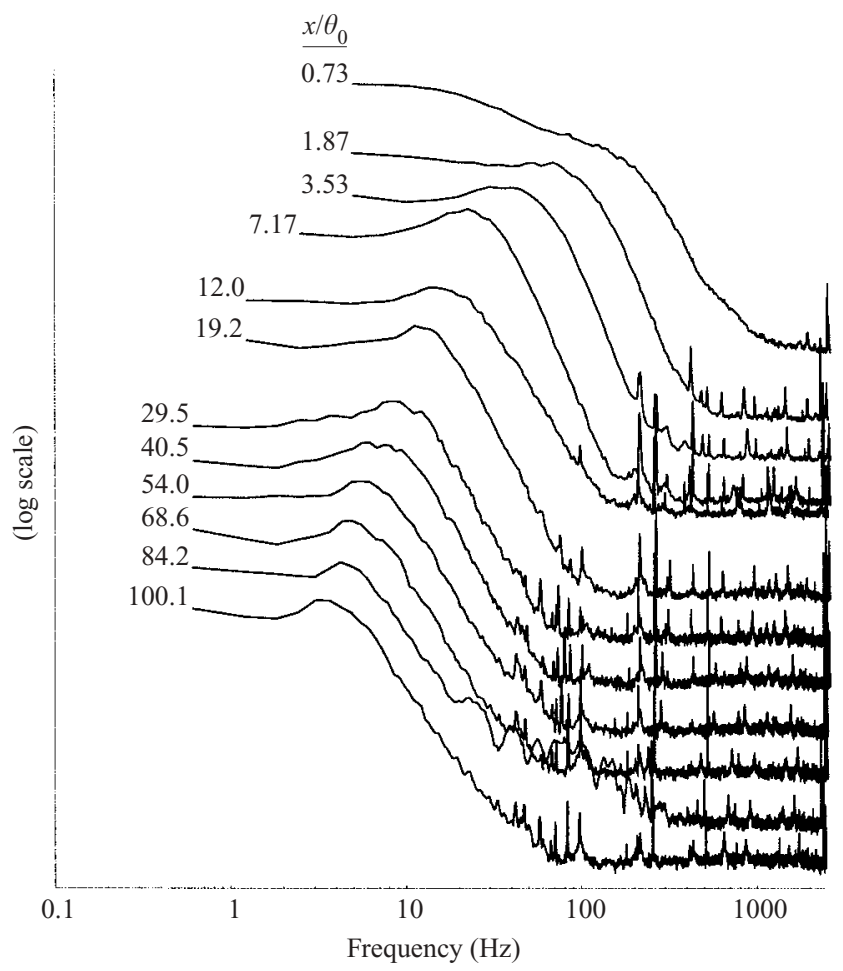

FIGURE 17 . Velocity spectra of entrainment for the 12 streamwise locations measured.

the mean streamwise velocity can be written as

$$
\bar{q}(y)=\int_{0}^{y} \bar{\omega} \mathrm{d} y .
$$

As described above, the convective velocity of the first instability was found to be $0.27 U_{0}$ from figure 16 near $x=0$. The 'scale' of velocity in this context $\left(2 U_{c}=0.54 U_{0}\right)$ can be interpreted as an 'apparent free stream' speed as observed by the vorticity filaments which participate in the first instability. The location where $\bar{q} / U_{0}=0.54$ was found to be $y^{+} \approx 42$ at $x=0$, see figure 3 . Evaluating equation (3) at $y^{+}=42$ shows that $54 \%$ of the time-averaged vorticity which separates from the boundary layer participates in the first instability. The increase in the coherent-motion convection velocity shown in figure 16 indicates that an increasing amount of the time-averaged vorticity from the boundary layer participates in the coherent motions as the flow evolves in the streamwise direction.

\subsection{Spectral properties of the entrainment stream}

The spectral properties of the entrainment stream in proximity to the active shear layer were used to investigate the coherent motions of the sub-shear layer region. The power spectral density (the Fourier transform of the autocorrelation) was computed for a single lateral $(y)$ location at each of the $12(x>0)$ streamwise locations identified previously in figure 8 . The lateral positions where $\tilde{q}(y) \approx 0.015 U_{0}$ were selected on the low-speed side where the velocity fluctuations appeared irrotational. These data are shown in $\log -\log$ format in figure 17. The amplitude of each data set was shifted vertically in order to distinguish the data sets on one figure. These data show a 


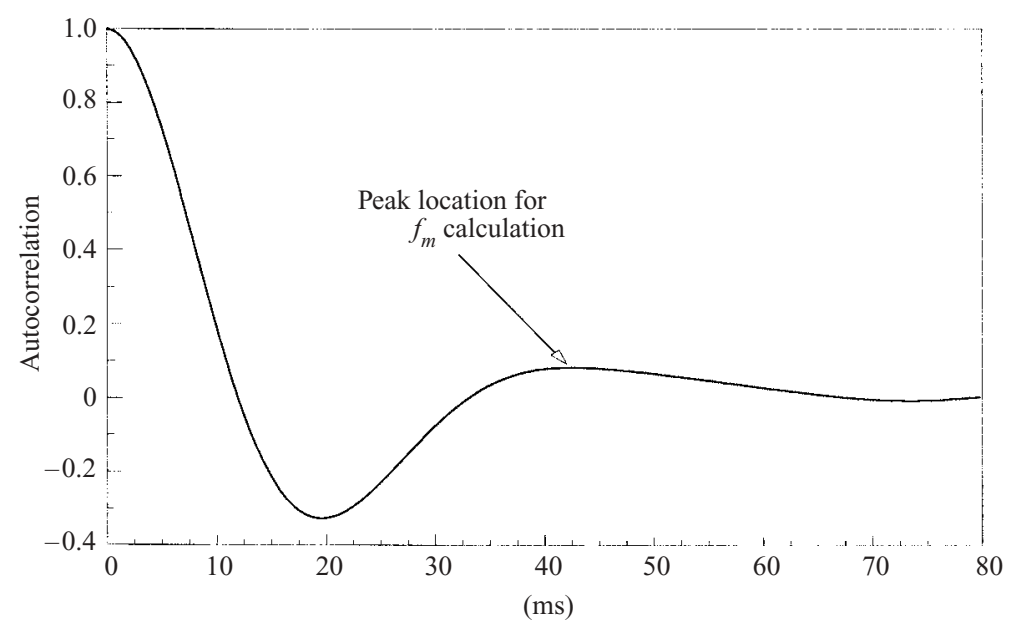

FIGURE 18. Sample of the autocorrelation function in the entrainment stream at $x / \theta_{0}=7.17$.

distinct local maximum, or 'hump', in the spectrum for all streamwise locations with the exception $x / \theta_{0}=0.73$. For example, the hump at $x / \theta_{0}=29.5$ occurs at $10 \mathrm{~Hz}$. Nearly identical results for the range $37<x / \theta_{0}<4800$ can be found in Hussain \& Zaman (1985, hereafter referred to as $\mathrm{H}-\mathrm{Z}$ ). It can also be observed that the frequency at which the local maximum occurs, $f_{m}$, decreases with downstream distance. This frequency can also be identified using the first peak of the autocorrelation function. This leads to a similar, although not necessarily the same, identification of a preferred frequency. Figure 18 shows an example of the autocorrelation function for $x / \theta_{0}=7.17$. It should be noted that at all locations, the 'hump' does not imply that the signal is periodic. These humps should be interpreted only as a preferred frequency at that streamwise location.

The frequency values $f_{m}$ (normalized by $\theta_{0}$ and $U_{0}$ ) were calculated from both the spectra and autocorrelations for the region $1.8<x / \theta_{0}<650$; see figure 19 . The frequency data from $\mathrm{H}-\mathrm{Z}$ in the region $37<x / \theta_{0}<4800$ (taken from figure 5 of that reference) are also shown in figure 19. Note that the boundary layer Reynolds number at separation was $R e_{\theta}=428$ in $\mathrm{H}-\mathrm{Z}$. The agreement is quite good in the overlap region between these data sets, which indicates that the dimensionless frequency at these $x / \theta_{0}$ locations is not a strong function of the Reynolds number of the separating boundary layer.

The frequency data can also be made non-dimensional using the free-stream velocity and the local momentum thickness. That is, the $f_{m}$ and the $\theta(x)$ values can be combined to form a new variable defined as $f^{*}=f_{m} \theta(x) / U_{0}$. These data are shown in figure 20. The $f^{*}$ value measured at the location $x / \theta_{0}=600$ is also shown on the figure. The functional dependence of $f^{*}$ can best be interpreted by considering the functional dependence of both the momentum thickness and the frequency from the respective curve fits of the data. Although the momentum thickness does not grow linearly in the region very near separation, all streamwise locations shown in figure 13 can be roughly approximated by

$$
\frac{\theta(x)}{\theta_{0}}=0.0312\left(\frac{x}{\theta_{0}}\right)+1.079 .
$$




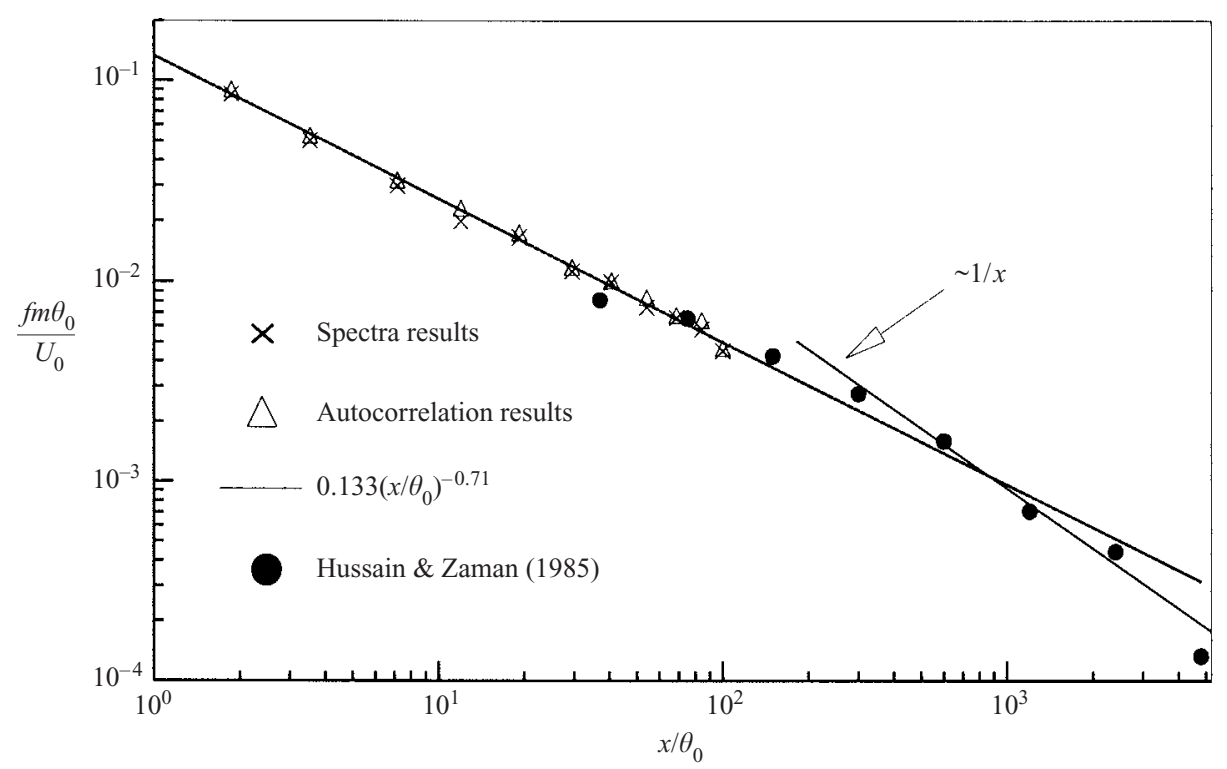

FIGURE 19. Streamwise variation in peak frequency.

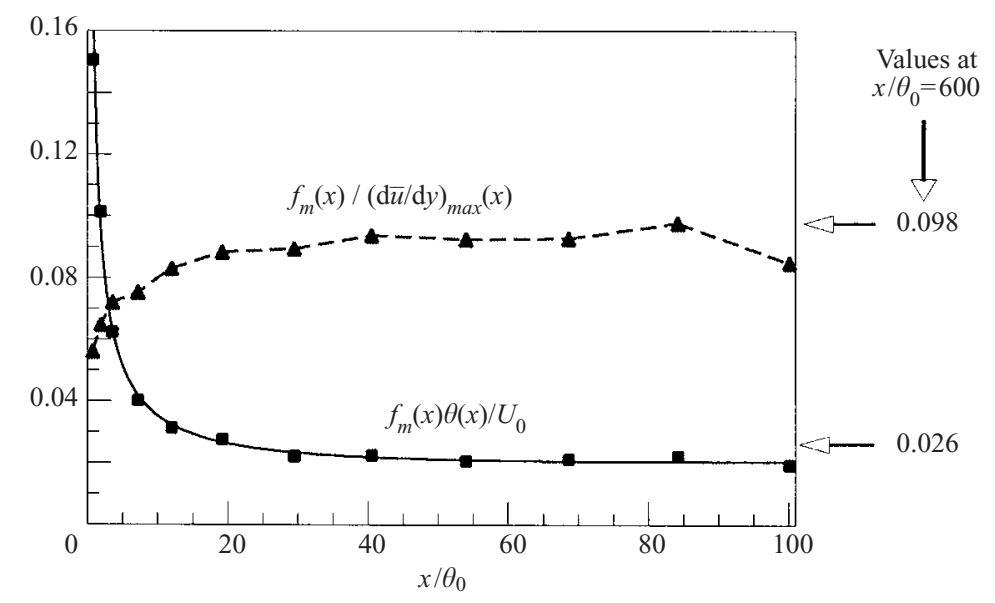

FIGURE 20. Non-dimensional frequency vs. $x / \theta_{0}$.

A fit of the frequency data is represented by

$$
\frac{f_{m} \theta_{0}}{U_{0}}=\frac{0.133}{\left(x / \theta_{0}\right)^{0.714}} .
$$

Therefore, the dimensionless frequency becomes

$$
f^{*}(x)=\frac{f_{m} \theta(x)}{U_{0}}=0.0041\left(x / \theta_{0}\right)^{0.286}+0.143\left(x / \theta_{0}\right)^{-0.714} .
$$

Equation (6) is shown as the solid curve on figure 20. The two terms of equation (6) represents two limiting regions of interest: the region very near separation, and the behaviour for large $x$. The larger values of $x / \theta_{0}$ are clearly dominated by the first term of equation (6). This equation would predict an $f^{*}$ which is slowly increasing for 
large $x / \theta_{0}$ values because the momentum thickness grows linearly, and the exponent of the frequency dependence is -0.71 . This is in contrast to the expected result that the dimensionless frequency would be constant. It is also noted that the largest downstream distance measurement location provided $f^{*}\left(x / \theta_{0}=600\right)=0.026$. This is somewhat $(25 \%)$ larger than the value of 0.021 from the $x / \theta_{0}<100$ data, although this difference is close to the uncertainty of the measurement. Note also that H-Z measured a value of 0.024 , although the uncertainty in those measurements seems to be considerably greater given the scatter in the H-Z data.

The $x$ dependence of $f^{*}$ has two possible explanations: either the $f^{*}$ values are streamwise dependent for all $x$, or the shear layer is still 'relaxing' from the initial upstream boundary conditions (i.e. the turbulent boundary layer). The second of these explanations is likely to be the correct one. It can be observed from the H-Z data in figure 19 that $a f_{m} \sim 1 / x$ dependence approximates the data reasonably well for $x / \theta_{0}>1000$, whereas $f_{m} \sim 1 / x^{0.71}$, as measured in the present data, seems to correlate well with the H-Z data for $x / \theta_{0}<1000$. It is possible that the present shear layer would also tend towards a $1 / x$ dependence for larger $x$ values given the agreement with the H-Z data in the region where the data overlap.

The non-dimensional frequency in the limit of small $x$ values is also of interest, particularly with regard to the sub-shear layer described in the previous sections. In terms of equation (6), the second term dominates the magnitude of $f^{*}$ for small $x / \theta_{0}$. This is because the frequency increases as $x$ is decreased, and the momentum thickness $\theta(x)$ approaches the value at separation $\left(\theta_{0}\right)$. A preferred scaling might be obtained by relating the frequency to the local maximum velocity gradient: $f_{\text {grad }}^{*}(x)=f_{m} /(\mathrm{d} u / \mathrm{d} y)_{\max }$. Note that this definition is equivalent to using the "vorticity thickness' scaling. These data are also shown on figure 20. This definition was motivated by the fact that both variables used in the definition of $f_{\text {grad }}^{*}(x)$ follow a power law $\sim x^{-n}$ as shown in figures 14 and 19. It can also be observed that the exponent of the maximum velocity gradient, $n=0.79$, closely matches the value 0.71 measured for the preferred frequency.

\subsection{The initial instability}

The frequency of the initial instability has been the focus of numerous studies (see e.g. Ho \& Huerre 1984). The understanding of how the boundary layer vorticity is redistributed downstream of separation is of considerable interest both fundamentally and technologically. Manipulation and periodic forcing of the boundary layer at separation can take particular advantage of the first instability in order to control the unsteady dynamics of the shear layer.

The near-separation region of a laminar shear layer is known to be unstable to small-amplitude perturbations as described by the Kelvin-Helmholtz instability. Linear stability theory has been used by many authors to understand and predict the frequency and growth rate of the first instability. There are several important assumptions of the linear theory which often include parallel flow, laminar flow with infinitesimal disturbances, and a specific velocity profile such as a hyperbolic-tangent function. Although these assumptions are not physically realized in any turbulent shear flow, the theory predicts the frequency of the fundamental instability for various geometries with marked success. The efficacy of this theory for shear layers and jets was reviewed by Ho \& Huerre (1984) and Thomas (1991). The Strouhal number of the most amplified frequency is defined as $S t_{0}=f_{m} \theta_{0} / U_{0}$. Note that some references use the average velocity in the case of a two-stream shear layer. This would change 


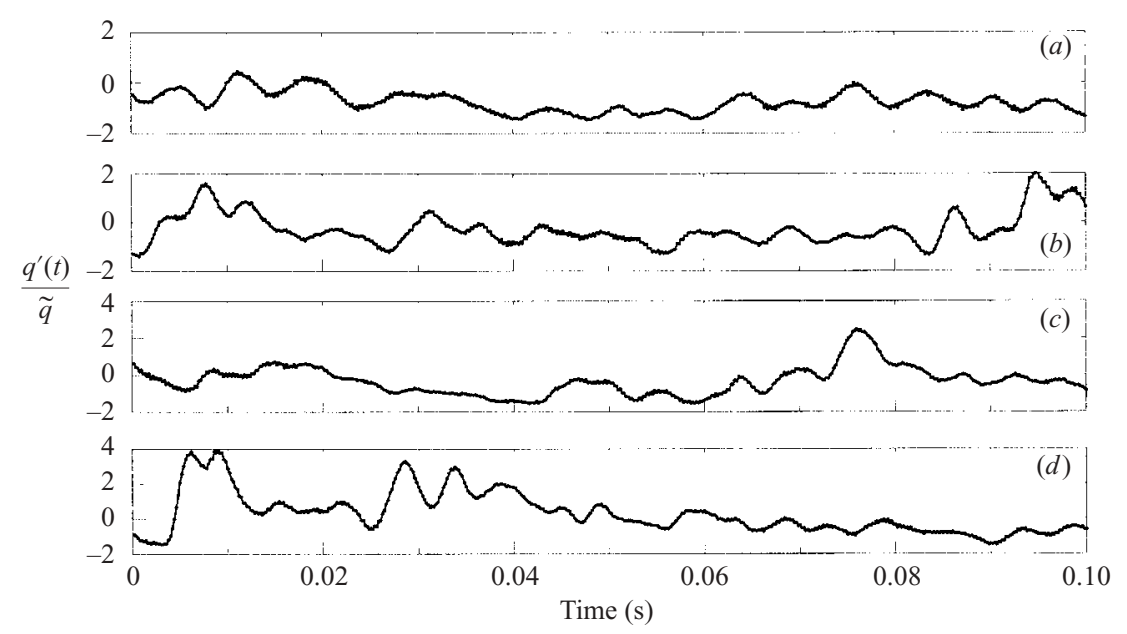

Figure 21. Time series samples from $x / \theta_{0}=0.73$.

the definition by a factor of two; the referenced values have been divided by two where appropriate for comparison to single-stream results.

In contrast, the effects of a turbulent boundary layer state and the Reynolds number on the initial instability have not been extensively investigated. Although the initial development region for laminar flow has received considerable attention, most shear layer studies which have a turbulent boundary layer do not consider the initial instability of the shear layer. Ho \& Huerre (1984) comment that the most amplified Strouhal number changes from 0.016 for a laminar flow to $0.022-0.024$ for turbulent flow as a result of 'presently unexplained reasons'. The remainder of this section will clarify their observation in terms of the sub-shear layer phenomenon.

The single-sensor hot-wire data described in the previous sections were used to observe the frequency of the initial instability. Using the spectral information shown in figure 17 it can be observed that the time-averaged spectra of velocity show a local maximum at the streamwise locations $x / \theta_{0} \geqslant 1.87$. Although these time serics are not periodic, the local maximum is interpreted to represent the preferred frequency. However, the lack of a local maximum in the spectra for $x / \theta_{0}=0.73$ does not imply that a preferred frequency does not exist. The flow visualization shown in figure 6 indicates that the first instability occurs in this region. This has motivated a more detailed examination of the time series data at this location to identify if a preferred frequency can be identified.

Several segments of time series data are shown in figure 21. These data show that pseudo-periodic motions do exist, although the frequency, phase and amplitude vary considerably during a relatively short time period. This explains why the spectra failed to show a local maximum value at a particular frequency. This result is also physically reasonable given that the vorticity convected from the viscous region of the boundary layer is highly unsteady and highly perturbed by the turbulence in the 'log region'. An alternative approach to identifying a preferred frequency was taken, using only short segments of the times series. Specifically, the time series data were divided into 0.1 and 0.2 segments for analysis. The autocorrelation was calculated for each of these segments. Several examples of these correlations are shown in figure 22 along with the autocorrelation of the full time record. The advantage of using the short time autocorrelations is that a distinct local maximum occurred in many of 


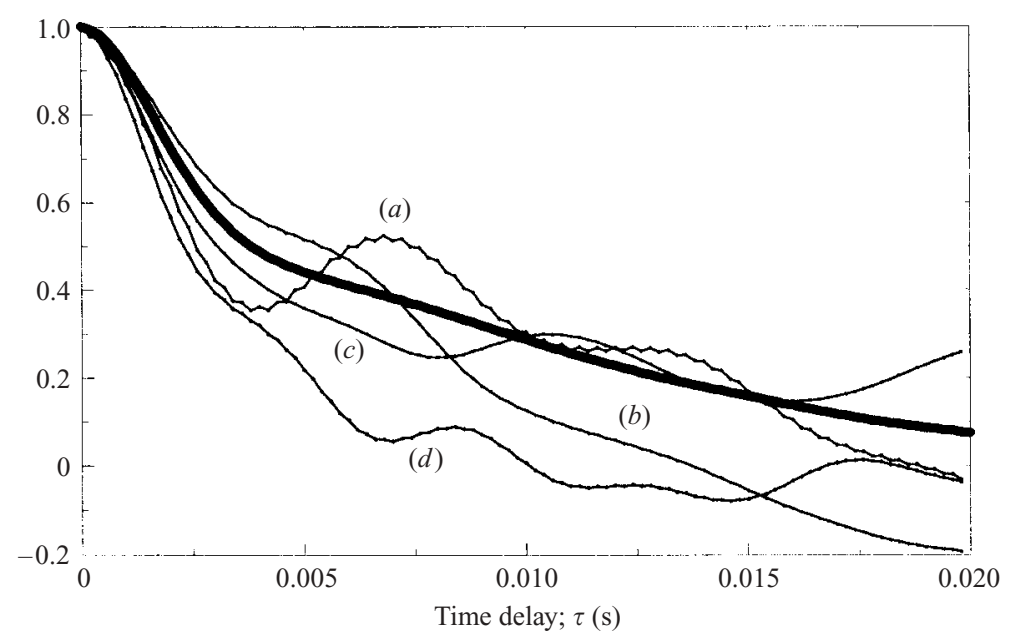

FIGURE 22. Autocorrelations of the time series at $x / \theta_{0}=0.73$. The long time averaged (thick line) as well as $0.1 \mathrm{~s}$ averages from the time series in figure 21 are shown.

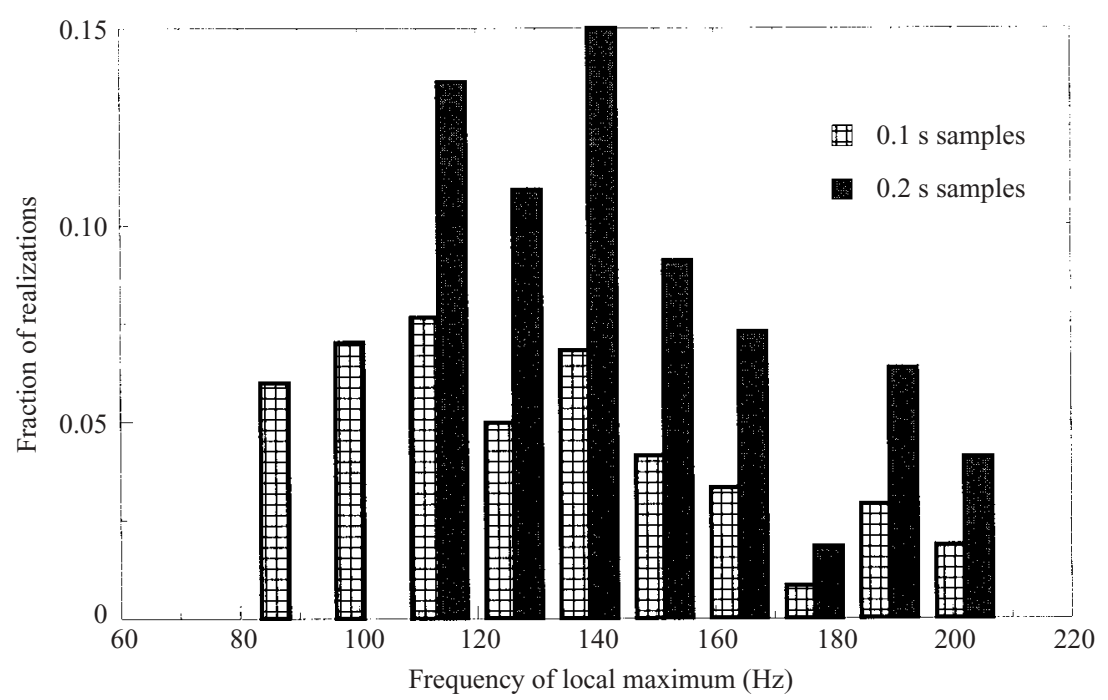

FIGURE 23. Histogram of observed frequencies from short time autocorrelations.

the calculations, indicating a preferred frequency during that time segment. Local maxima were found in $51 \%$ of the $0.1 \mathrm{~s}$ time series and $37 \%$ of the $0.2 \mathrm{~s}$ time series. A histogram of the observed frequencies is shown in figure 23 . The mean frequency from the $0.1 \mathrm{~s}$ sampled was $130 \mathrm{~Hz}$. The mean value from the $0.2 \mathrm{~s}$ samples was $146 \mathrm{~Hz}$. These values are in nominal agreement with the value of $124 \mathrm{~Hz}$ obtained by extrapolating the curve fit given by equation (5). This agreement adds confidence that the coherent motions observed in the flow visualization at the location $x / \theta_{0}=0.73$ do exhibit a preferred frequency.

The next step in the determination of $S t_{0}$ is to choose velocity and length scales to non-dimensionalize the indicated frequency. The standard choice of $U_{0}$ and $\theta_{0}$ would not be appropriate since it is only the near-wall region that is participating in this 


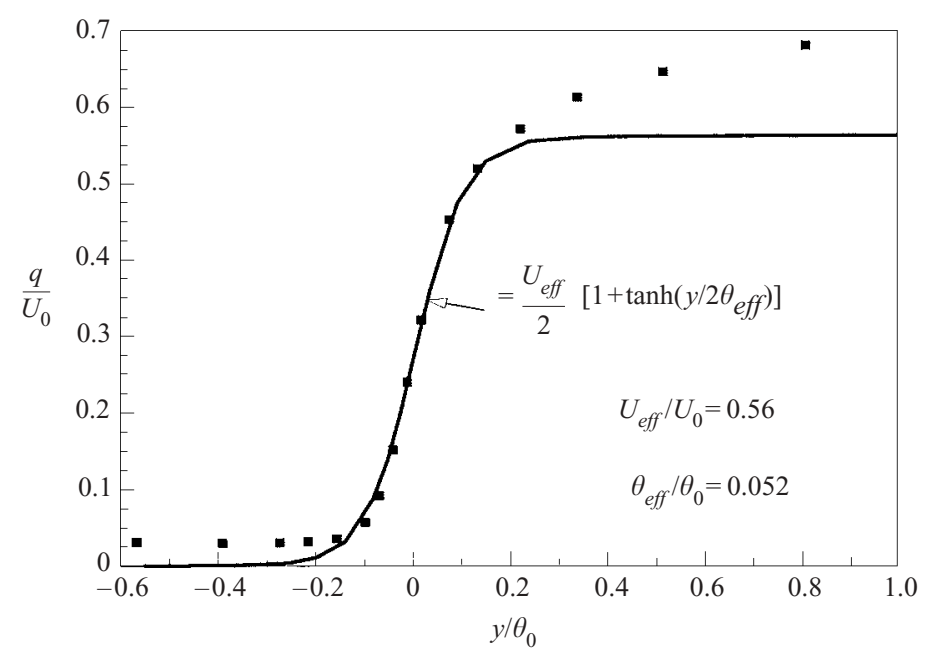

FIGURE 24. Comparison between tanh function shown and the velocity data at $x / \theta_{0}=0.73$.

first instability. It seems illogical to use, for example, the boundary layer momentum thickness $\left(\theta_{0}\right)$ to scale a phenomenon which does not involve the entire boundary layer. The new scales of velocity and length will be $U_{\text {eff }}$ and $\theta_{\text {eff }}$ to imply that it is the effective velocity scale and the effective momentum thickness only that lead to the first instability and the formation of the first coherent motions.

A logical choice for the velocity scale is $U_{\text {eff }}=0.54 U_{0}$. This is justified by the apparent convection velocity of the first instability shown in figure 16 . The effective momentum thickness of the sub-shear layer was determined by fitting the hyperbolic tangent profile to the mean flow measurements acquired at $x / \theta_{0}=0.73$. The data were fit to the equation

$$
\frac{q(y)}{U_{0}}=\frac{U_{\text {eff }}}{2}\left[1+\tanh \left(\frac{y}{2 \theta_{\text {eff }}}\right)\right]
$$

in the range $0<\bar{q}<0.54 U_{0}$. The mean velocity data and equation (7) are in good agreement over this range, which corresponds to $\left|y / \theta_{0}\right|<0.1$; see figure 24 . Equation (7) was used to find $\theta_{\text {eff }}$ because the linear stability theory calculation of the most amplified wavenumber is derived from the tanh profile. The effective momentum thickness was determined to be $\theta_{\text {eff }}=0.5 \mathrm{~mm}\left(\theta_{0} / \theta_{\text {eff }}=19.2\right)$ by a leastsquares fit between the data points and equation (7). Finally, the values $f_{m}=130 \mathrm{~Hz}$, $U_{\text {eff }}=0.54 U_{0}=3.98 \mathrm{~m} \mathrm{~s}^{-1}$, and $\theta_{\text {eff }}=0.5 \mathrm{~mm}$ lead to $S t_{0}=0.0163$. Although the uncertainty of this measurement is estimated to be $\pm 10 \%$, this value is in excellent agreement with both linear theory and all existing literature for shear layers originating from laminar boundary layers.

Given the above observations, a more objective scaling of the initial frequency could be used for turbulent separation. Specifically, the realization that only the near-wall vorticity participates in the first instability implies that the wall scaling could be used. That is, using the viscous wall units defined for the boundary layer upstream of separation, the dimensionless frequency can be written as $S t_{0}^{+}=f_{0} v / u_{\tau}^{2}$. For the present data, $S t_{0}^{+}=0.025$. It remains a question of interest if this remains constant at other turbulent Reynolds numbers. It is noted that the value computed (from the Blasius solution) for laminar separation is $S t_{0}^{+}=0.075$. 


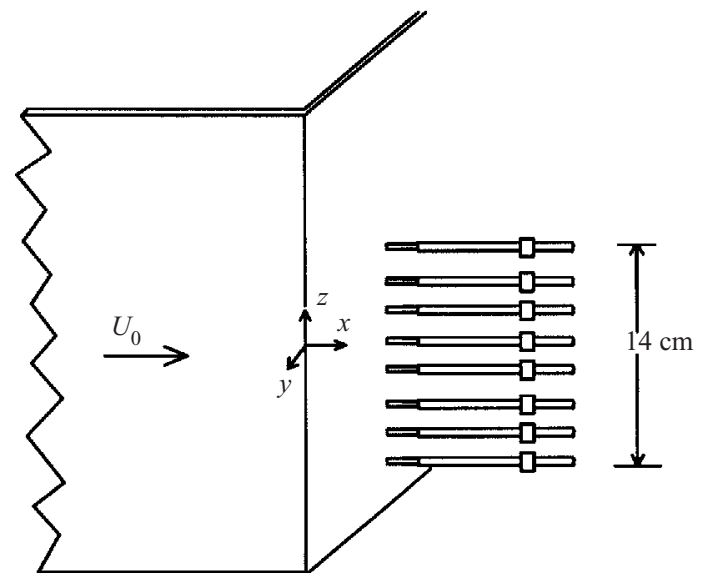

FIGURE 25. Schematic of eight-wire rake in the shear layer.

\section{Experiment 4: spanwise correlation measurements}

The statistical data presented thus far were acquired at a single spanwise location. Because the flow is stochastically homogeneous, these data are representative of the entire flow field excluding the sidewall boundary layers $\left(z / \theta_{0} \approx \pm 90\right)$. In addition to these data, it is of interest to document the multi-point spanwise correlation of the velocity field. This interest originated from the velocity measurements taken on the entrainment side of the shear layer which indicated that motions that exists within the shear layer have sufficient coherence to be detected well into the irrotational flow. The spanwise extent of these coherent motions is of considerable importance in applications involving acoustics, fluid-structure interactions, etc.

The data were acquired using a rake of eight single-wire sensors aligned in the $z$-direction. The sensors were equally spaced at $\Delta z / \theta_{0}=2.05$. A schematic of the rake and its orientation is shown in figure 25 . These probes were traversed through the near-separation region using the same $(x, y)$ locations as those shown in figure 8 . The time series data were recorded for $60 \mathrm{~s}\left(t U_{0} / \theta_{0}=4.4 \times 10^{4}\right)$ at a data acquisition rate of $5000 \mathrm{~Hz}$ at each spatial location.

The presentation of these results is divided into the two following sub-sections. First, several short time segments of velocity from the low-speed side will be presented. These data will help to orient the reader to the data acquisition procedures and they will assist in the interpretation of the results. Secondly, data will be provided from calculations of the zero-time-delay cross-correlation for the $(x, y, \Delta z)$ positions measured.

\subsection{Example realizations in the irrotational stream}

Four streamwise locations were selected to provide example realizations from the eight-wire rake: $x / \theta_{0}=3.53,12.0,40.5$, and 84.2 . The velocity fluctuations are shown as contour plots in figure $26(a-d)$. The abscissa represents the time elapsed from an arbitrary starting point (i.e. when the A/D system was activated) and was not intended to isolate any specific observed features. The time was non-dimensionalized using the local value of the peak frequency as shown in figure 17 ; that is, $\lambda \equiv($ time $) \times f_{m}$ which represents the data in terms of the integer number of averaged wave periods. Ten wave periods are shown in these figures. Longer time records yield qualitatively similar representations of the flow field. The contours show the fluctuating component of velocity from all eight sensors normalized by the standard deviation of the respective signals. The legend for the grey scale shows that light shading indicates positive 


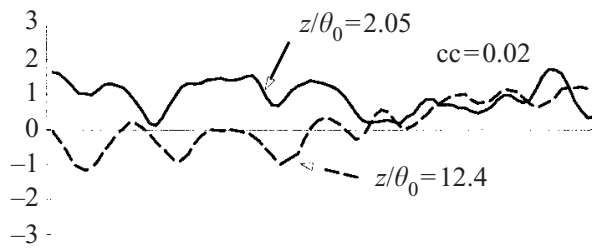

(a)
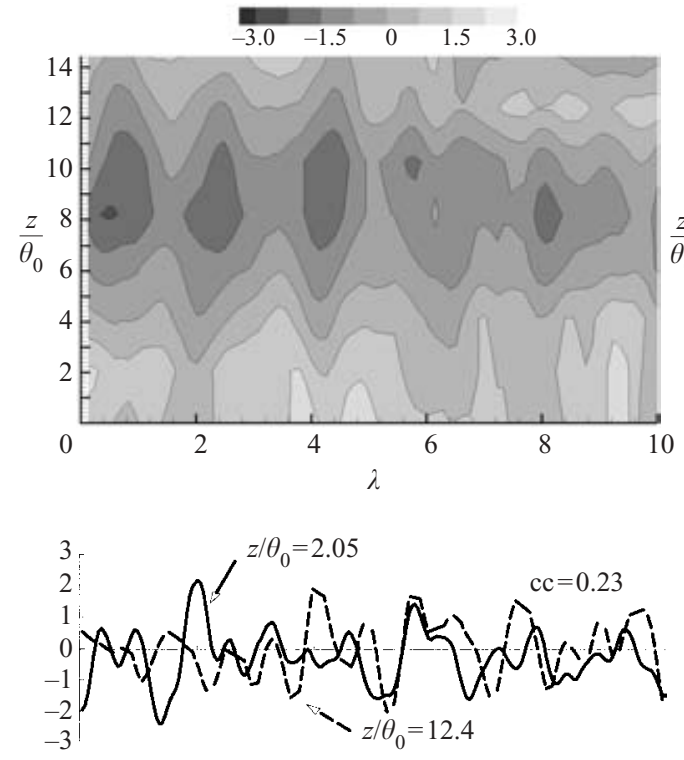

(b)

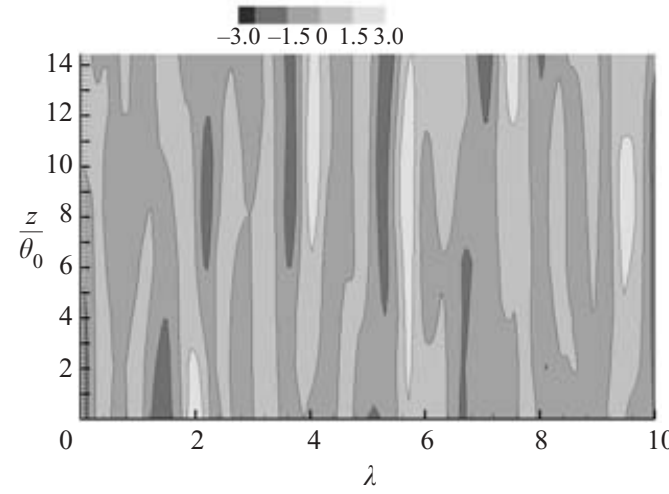

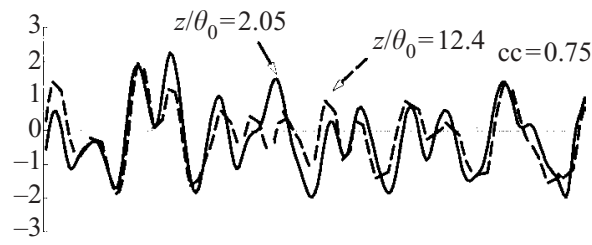

(c)
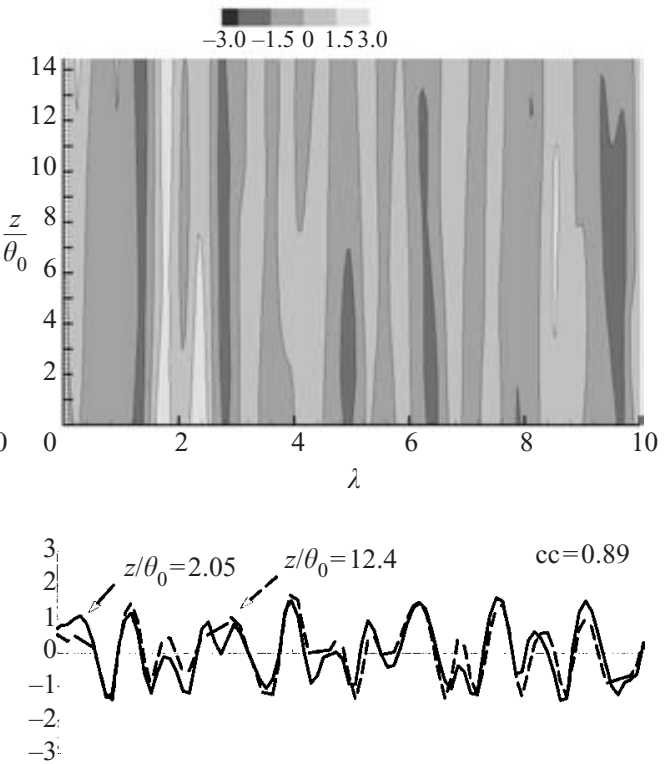

(d)

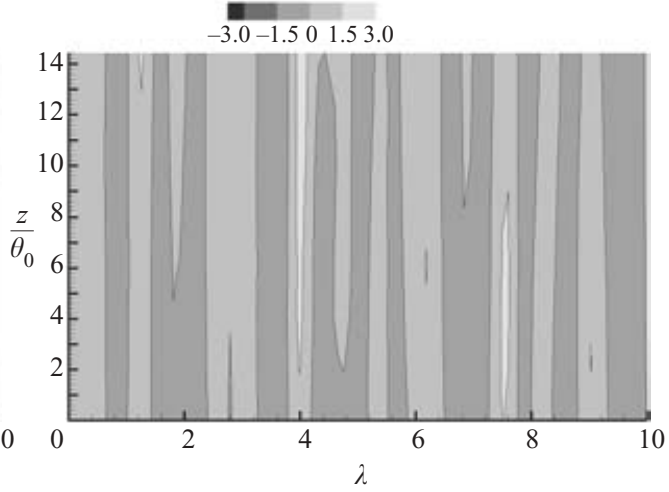

FIGURE 26. Contour plots show magnitude of velocity fluctuations from eight sensors normalized by the standard deviation of the respective velocity fluctuations. The contours are interpolated between the eight discrete locations. The upper $x, y$ plots show time traces taken from wires located at $z / \theta_{0}=2.05$ and 12.4 for the same time period. The correlation coefficient for these two $q(t)$ signals from the full time record is indicated by 'cc'. (a) $x / \theta_{0}=3.53, y / \theta_{0}=-3.7 ;$ (b) $x / \theta_{0}=12.0, y / \theta_{0}=-5.37 ;(c) x / \theta_{0}=40.5, y / \theta_{0}=-10.9$; (d) $x / \theta_{0}=84.2, y / \theta_{0}=-19.6$.

fluctuations in velocity, and dark shading negative fluctuations. For each of the contour plots, the time traces from the wires located at $z / \theta_{0}=2.05$ and 12.4 are shown for comparison.

Growth in the spanwise coherence of the entrainment velocity was observed as $x / \theta_{0}$ was increased from 3.53 to 84.2. Specifically, figure 26(a) shows clear evidence that 


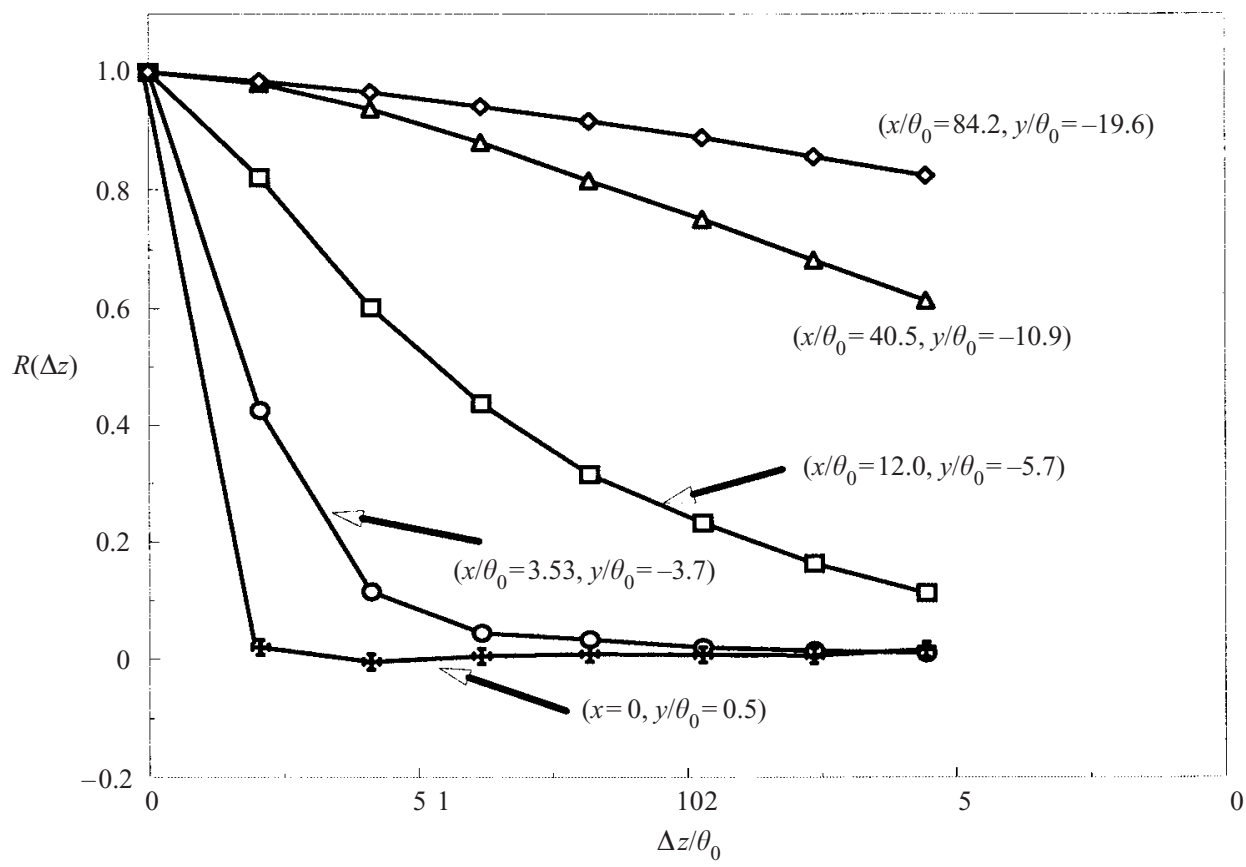

FiguRE 27. Spanwise correlation coefficient of the data shown in figure 26.

the motions which led to these fluctuations do exhibit a coherence in the spanwise direction, although the spatial extent is limited. Figure 26(b) could be described similarly, although the spatial extent of the coherent motions has obviously 'grown'. It is instructive to reiterate the idea of the sub-shear layer when considering these spanwise correlations. As a specific example, recall that at the streamwise location $x / \theta_{0}=12$, the flow field for $y / \theta_{0}>2$ is stochastically identical to the boundary layer. Therefore, the spanwise correlation of the motions is observed to be several times larger than the local sub-shear layer thickness.

In order to directly contrast this large spanwise coherence with the boundary layer flow, the correlation coefficient for these four locations as well as one position within the boundary layer (at $x=0, y=0.5 \theta_{0}$ ) are shown in figure 27. It is clear from these measurements that the larger spanwise coherence is developed downstream of the separation point. The correlation values are considerably larger in the low-speed region near the sub-shear layer than in the boundary layer, even at small $x / \theta_{0}$ values.

The continued growth in coherence is also seen in figures $26(c)$ and $26(d)$, where the irrotational fluid motion approaches that of a two-dimensional flow. These measurements are similar to those of Bowand \& Trout $(1980,1985)$. Twelve wires were placed in the low-speed side of a two-stream shear layer facility in the Browand \& Trout investigation. These authors observed coherent velocity fluctuations in the irrotational flow near to the active shear layer, with a correlation length scale that increased in the streamwise direction. Also, the short time realizations of the velocity fluctuations shown in Browand \& Trout are qualitatively similar to those shown in figure 26.

\subsection{Correlation coefficient data}

The correlation coefficient $R(\Delta z)$ was calculated (as shown in figure 27) for each of the $(x, y)$ locations (see figure 8 for the $x, y$ data points used). The domain of the data set can be represented by the 'volume' of points $(x, y, \Delta z)$. The visualization of 

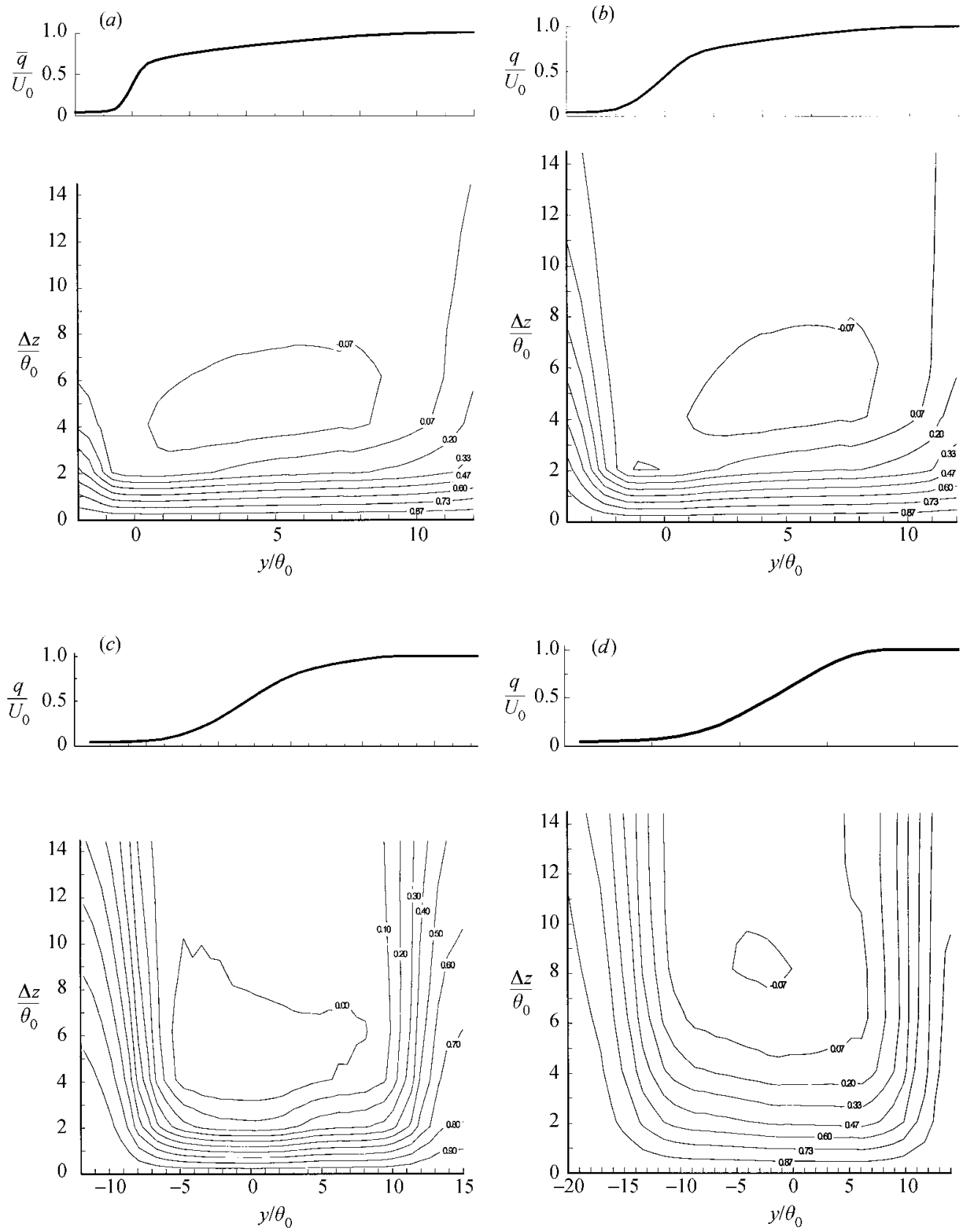

FIGURE 28. Spanwise correlation field at $(a) x / \theta_{0}=3.53,(b) 12.0,(c) 40.6,(d)$ 87.2. The contours represent the magnitude of $\overline{\bar{q}^{\prime}(x, y, z) q^{\prime}(x, y, z+\Delta z)} / \overline{\left(q^{\prime 2}\right)}$.

these data was made possible by 'slicing' the volume in planes perpendicular to either the $x$ - or the $z$-direction. First, the streamwise locations $x / \theta_{0}=3.53,12.0,40.5$, and 84.2 (i.e. the positions used in figure 26) were selected. Figure 28( $a-d)$ shows contours of the correlation coefficient at these locations. The mean velocity profile is included with each of the figures for reference. Figure $29(a-d)$ shows contours of $R$ at specific 

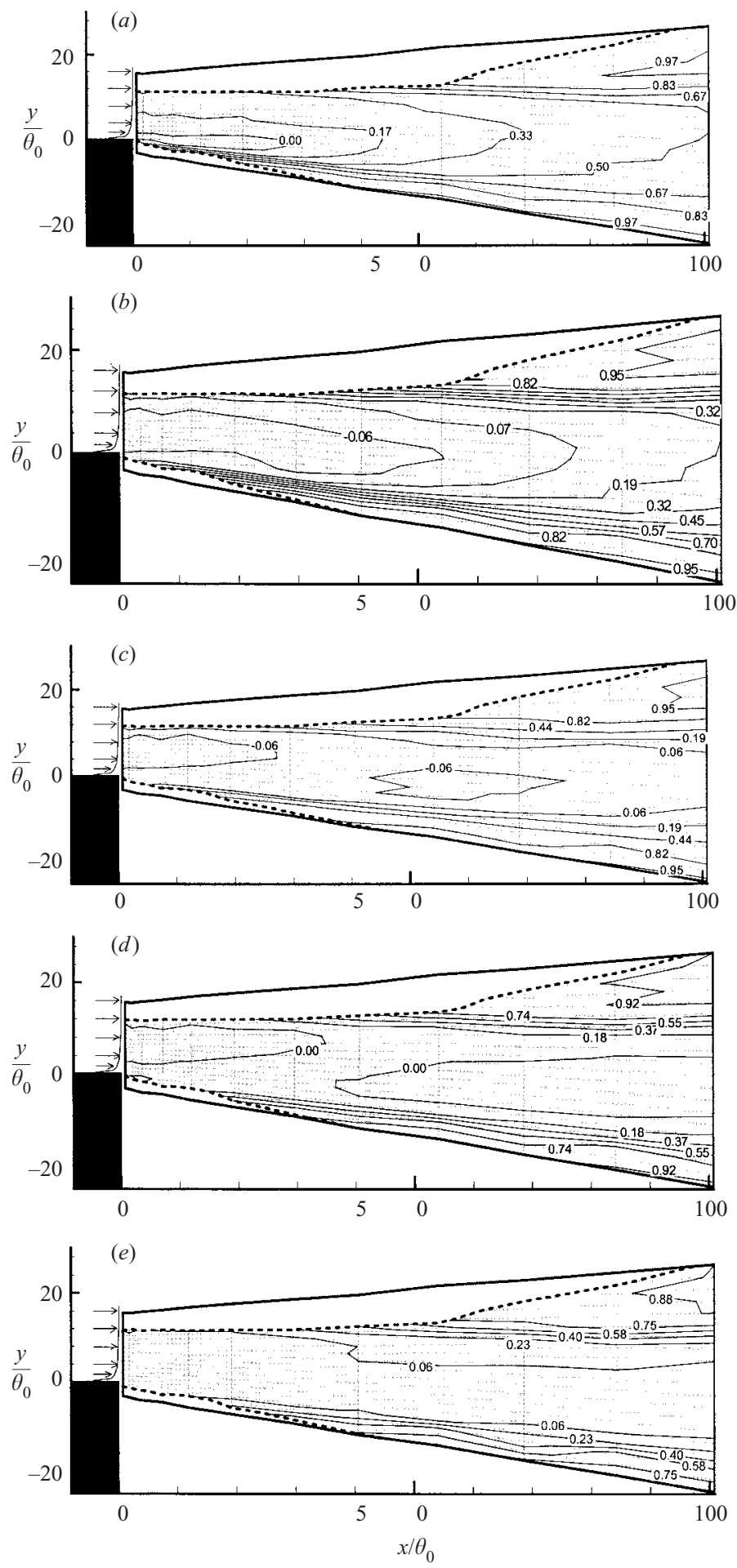

Figure 29. Correlation coefficient (as defined in figure 28) at $(a) \Delta z / \theta_{0}=2.08,(b) 4.16$, (c) $6.24,(d) 8.32,(e) 14.5$. Note that the solid boundary represents the spatial extent of the measurements. The dashed boundary represents the region where $u_{r m s} / U_{0}>0.01$. The measurement locations within this domain are identified by the grid. A schematic of the boundary layer mean profile near $x=y=0$ is shown for reference. 
$\Delta z$ values. The solid boundary surrounding the contour plot represents the spatial extent of the data acquisition. The dashed boundary represents the contour line where the fluctuation levels are $1 \%$ of the free-stream velocity (see figure 11). Correlation data are shown in the region where the standard deviation of the fluctuations exceeds $1 \%$ of $U_{0}$.

Several important features of the flow field can be observed from these measurements. First, the central region of the shear layer shows quite low spanwise correlation values. This is in contrast to the low-speed irrotational flow measurements which exhibit a large correlation length with respect to the local length scales. Although it is apparent that the irrotational fluctuations are caused by the motions existing in the turbulent fluid, the correlation magnitudes are diminished by the presence of relatively large fluctuating velocities that can be attributed to smalllength-scale motions.

A second feature observed from these data is the difference between the high- and low-speed sides of the shear layer. Specifically, the correlation length on the low-speed side grows smoothly and continuously from the separation point, whereas the highspeed side shows an abrupt chage in correlation values near $x / \theta_{0}=55$. These data reinforce the inferred presence of the sub-shear layer, and they also provide insight into how the sub-shear layer evolves into a fully developed shear layer. Specifically, the vortical fluid which occupied the outer region of the boundary layer does not participate in the instability which leads to the large-scale spanwise motions. At increasing streamwise positions, additional vortical fluid is drawn in to 'participate' with the coherent motions. The dramatic change in the correlation field near $x / \theta_{0} \approx 55$ indicates that the motions have become strong enough to have a measurable effect on the high-speed irrotational fluid. It is instructive that the time-averaged convection velocity of the coherent motions at this location is approximately $82 \%$ of the final value of $0.5 U_{0}$ (see figure 16).

\section{Conclusions with discussion}

The experimental data described in the previous sections lead to a number of observations about the transition from an attached boundary layer to a single-stream shear layer. The significant difference between the present study and previous research is the moderately high Reynolds number at separation $\left(R e_{\theta}=4650\right)$, and a relatively large integral length scale $\left(\theta_{0}=9.6 \mathrm{~mm}\right)$ which allowed excellent spatial resolution of the measurements. In this section each main conclusion is listed, followed by a discussion and interpretation of the relevant data sets.

(i) The stochastic properties of the boundary layer at separation $(x=0)$ appear to be unaffected by the flow field downstream of separation.

This conclusion is supported by several of the experiments. First, the zero crosscorrelation magnitudes between velocities measured in the boundary layer and in the entrainment stream is a strong indication that a coupling does not exist between the two regions. The lack of correlation in the low-pass-filtered time series further supports this result. The reported stochastic properties of the boundary layer show that the moments of the velocity and vorticity fields are in good agreement with the existing literature on flat-plate boundary layers without separation. If the shear layer did have an effect on the boundary layer at separation, it was not apparent in any of the point statistics or correlation measurements.

The streamwise invariance of the statistics in the outer region of the boundary layer provides additional evidence to support this conclusion. In other words, not only do 
the shear layer fluctuations not affect the boundary layer at $x=0$, there is also no measurable effect on much of the $y>0$ region for a considerable length downstream. This will be discussed further in (ii) below.

The fact that a high-Reynolds-number boundary layer which separates at a $90^{\circ}$ edge is not affected by the separation is important for several reasons. If the separation region were to be modelled using a computational method, the upstream boundary conditions could be specified at $x=0$ by the stochastic properties of the approach boundary layer. If this conclusion were not supported by the observations, then the separated flow field would have been found to affect the stochastic properties upstream of separation, and the inlet conditions to the computation would have to be specified at some $x<0$, rather than at $x=0$.

It is likely that communication from the boundary layer to the shear layer is dependent on the Reynolds number. Laminar and low-Reynolds-number turbulent flows at separation are likely to be more sensitive to the perturbations caused by the downstream motions. A future study of this topic could attempt to measure an objective indication of the communication for a range of Reynolds numbers.

(ii) The outer region of the boundary layer is stochastically streamwise invariant for several integral lengths downstream of separation.

This streamwise invariance of the boundary layer properties is evident in all of the data identified above. For example, the plots showing statistics in figures $8-11$ and 29 all show invariant properties of the flow in the region $0<x / \theta_{0}<50$ at lateral positions of $y>0.15 x$. This adds information to a body of literature which exists on the topic of inner and outer scales in the boundary layer; see, for example Klewicki (1989), Marusic \& Perry (1990), and Hamelin \& Alving (1996). The present observations indicate that not only are the outer scales not dependent on the local production of near-wall turbulence, but that the flow is not even affected by the large-scale fluctuations of the shear layer measured in the $y \approx 0$ region.

(iii) The separation region can be described as a 'sub-shear layer' in which only the near-wall vorticity participates in the initial shear layer instability.

The region which defines the sub-shear layer is qualitatively evident in the flow visualization image shown in figure 6 . This may also be identified as the wedge-shaped region roughly defined by $u_{r m s} / U_{0}>0.087$ on figure 11 . Many of the salient features that are associated with canonical shear layers have been identified in this region. For example, it can also be observed that the 'spread angle' of the sub-shear layer is equal to that of the fully developed shear layer. This can be quantified from the two contour lines for which $u_{r m s} / U_{0}=0.087$ on figure 11 . The normalized distance between these lines, say, $\Delta y$, can be expressed as $\Delta y / x$. This was found to be $0.17 \pm 0.01$ for $5<x / \theta_{0}<650$. That is, the spread angle is the same in the sub-shear layer and the fully developed shear layer.

An important feature of shear layers is the presence of coherent motions which develop from the inflectional instability mechanism (see Ho \& Huerre 1984 for a review). These motions are known to move in the streamwise direction with a well-defined convection velocity. In fully developed single-stream shear layers, this velocity is known to be nominally $2 U_{c} / U_{0}=1.0$. The convection velocity in the sub-shear layer region is shown in figure 16 . These data indicate a dramatic rise in the convection velocity from $2 U_{c} / U_{0} \approx 0.54$ at separation to a value near 1.0 at $x / \theta_{0}=120$. It is inferred from these data that only the near-wall vorticity is 'rolling up' or participating in the inflectional instability. A quantitative estimate of the fluid domain which participates in the initial roll-up has been identified as that 
below the $\bar{q} / U_{0}=0.54$ isotach. Equivalently, this bound is given by $y^{+}<42$ for the present $\left(R e_{\theta}=4650\right)$ boundary layer. As the sub-shear layer grows into the outer part of the boundary layer, more of the vorticity is incorporated into the coherent motions thereby increasing both the width of the sub-shear layer and the convection velocity.

The present understanding of the vorticity field within a turbulent boundary layer is consistent with the current findings. That is, the stochastic and instantaneous properties of the motions which define the inner and outer scales of the boundary layer are significantly different. The near-wall motions are described by, for example Adrian, Meinhart \& Tomkins (2000), and Panton (2001). In these references, the near-wall region is understood to be composed of groups of coherent motions which define localized shear layers. These result in 'vortex packets' which evolve into the outer scales of motion. It is intuitively reasonable that the near-wall region with a high level of organization of vorticity would be subject to inflectional instability immediately downstream of separation. In contrast, the motions farther from the wall are usually considered to be more disorganized and not closely correlated with the motions very near to the wall. The current measurements of spanwise vorticity fluctuations at $x=0$ support this viewpoint. It is therefore reasonable that these outer motions would not participate in the roll-up phenomenon.

(iv) The recognition of the sub-shear layer allows a physical explanation for why shear layers with a turbulent boundary layer at separation approach self-similarity at smaller $x / \theta_{0}$ values than shear layers with a laminar separation.

The streamwise location where the flow field becomes self-similar has been found to be dependent on the state and Reynolds number of the boundary layer at separation. For example, Bradshaw (1966) recommends a value of $x / \theta_{0}>1000$ for self-similarity given a laminar separation. Hussain \& Zaman (1985), Bruns (1990), and the present study have found that $x / \theta_{0}>100$ is sufficient for the mean velocity to exhibit selfpreservation when the separating boundary layer is turbulent.

These differences can be explained in terms of the length scales of the turbulent vs. laminar boundary layers. Dimotakis \& Brown (1976) suggested that it is not the downstream location as defined by a number of original integral length scales (e.g. $\left.x / \theta_{0}\right)$ that is important for the establishment of a self-similar condition. Rather, it is the number of 'interactions' between the large-scale motions of the flow. The number of interaction will depend on the ratio of the streamwise location to the spacing of the initial disturbance. Specifically, the number of interactions $m(x)$ is estimated (equation (14) of Dimotakis and Brown) to be

$$
m(x)=\log _{2}\left(x / l_{0}\right)
$$

where $l_{0}$ is the spacing of the initial disturbance. For a laminar boundary layer at separation, the criterion $x / \theta_{0}>1000$ is equivalent to a value of $m \approx 4$. In the present study the stream-wise location $x / \theta_{0}=100$ corresponds to a value of $m$ between 6 and 7 , given that the spacing of the initial disturbance was $l_{0} \approx \theta_{0}$. This small value of initial disturbance length compared with $\theta_{0}$ is a direct result of the presence of the sub-shear layer.

(v) The dimensionless frequency of the initial instability agrees with linear stability theory when appropriate effective velocity and length scales are identified.

The measurement and prediction of the dimensionless frequency of the initial stability has received considerable attention in the literature; see, for example, Ho \& Huerre (1984) and Thomas (1991). This focus is a result of the universal nature of the initial instability in a variety of shear layer and jet flow fields. Additionally, 
linear theory has been quite successful in the prediction of this dimensionless frequency for many experiments with laminar boundary layers at separation. However, differences in the measured frequencies have been found in shear layers with turbulent boundary layers at separation. The present study has shown that these differences are a result of the different length and velocity scales that exist in a turbulent boundary layer. Specifically, a laminar boundary layer can be fully characterized by the free-stream velocity and momentum thickness. In contrast, a turbulent boundary layer has outer scales (free-stream and momentum thickness) and inner scales $\left(u_{\tau}\right.$ and $\left.v / u_{\tau}\right)$. The data presented suggest that only the near-wall vortical fluid participates in the initial instability at this Reynolds number. It is then quite reasonable that the outer scales would not be functionally related to the measured frequencies.

(vi) Preferred frequency and spanwise correlation measurements have shown that the characteristic large-scale motions observed in self-similar single-and two-stream shear layers are present in the sub-shear layer region.

Several investigators have studied the properties and importance of the largest scales of motion in shear layers. These motions appear to have a large spanwise coherence and a significant fraction of the total turbulent kinetic energy of the flow. Experimentalists have sought to measure these features in order to better understand their role and dynamic significance; see, for example, Hussain \& Zaman (1985), Browand \& Weidman (1976), and Browand \& Trout (1980, 1985).

The present experiments have found that a number of the features measured in the self-similar regions of both two-stream and single-stream shear layers have also been observed in the sub-shear layer region. For example, the preferred frequency exhibited by both the sub-shear layer and self-similar shear layer can be described by the relatively simple equation: $f_{m} \sim x^{-0.71}$ (for $x / \theta_{0}>1$ ) as shown in figure 19 . It can be inferred that the physical mechanisms which lead to this preferred frequency are present throughout the flow field downstream of separation. Note that the exponent -0.71 is anomalous, in that the expected value from self-similarity is -1 . The reason for this is not clear, although Hussain \& Zaman (1985) show that an exponent of -1 is not observed until downstream distances of $x / \theta_{0}>300$.

A second feature observed in the sub-shear layer region was the large spanwise correlation values observed in the near-separation region. Specifically, figure $26(a)$ shows evidence of spatial organization at the location of the first instability $\left(x / \theta_{0} \approx 1\right)$. The correlations then grow in spanwise extent in the streamwise direction. These observations are consistent with observations made by Browand \& Trout $(1980,1985)$ in the self-similar region of a two-stream shear layer.

The observations noted above justify the terminology 'sub-shear layer'. Specifically, the region exhibits characteristics which are associated with self-similar shear layers, while the prefix 'sub' denotes that the shear layer is growing adjacent to what appears to be the outer region of a flat-plate boundary layer.

The single-stream shear layer facility was financially supported by the Ford Motor Company, and primarily designed and fabricated by the first author. The donation of the four entrainment fans from Aerotech, Inc. of Mason, MI is gratefully acknowledged. Partial support for a Graduate Research Assistantship to S. C. M. was also provided by the Office of Naval Research, grant number N00014-00-1-0753 to the University of Utah with MSU as a sub-contractor. The grant monitor was Dr L. Patrick Purtell. 


\section{REFERENCES}

Adrian, R. J., Meinhart, C. D. \& Tomkins, C. 2000 Vortex organization in the outer region of the turbulent boundary layer. J. Fluid Mech. 422, 1-53.

Ali, S. K., Klewicki, C. L., Disimile, P. J., Lawson, I. \& Foss, J. F. 1985 Entrainment region phenomena for a large plane shear layer. Proc. Fifth Symp. on Turbulent Shear Flows.

Bradshaw, P. 1966 The effect of initial conditions on the development of a free shear layer. J. Fluid Mech. 26, 225-236.

BRowand, F. K. \& LaTigo, B. O. 1979 Growth of the two-dimensional mixing layer from a turbulent and nonturbulent boundary layer. Phys. Fluids 22, 1101-1019.

Browand, F. K. \& Trout, T. R. 1980 A note on spanwise structure in the two-dimensional mixing layer. J. Fluid Mech. 117, 771-781.

Browand, F. K. \& Trout, T. R. 1985 The turbulent mixing layer: geometry of large vortices. J. Fluid Mech. 158, 489-509.

Browand, F. K. \& Weidman, P. D. 1976 Largest scales in the developing mixing layer. J. Fluid Mech. 76, 127-144.

BRUNS, J. 1990 Evaluation of hot-wire velocity and transverse vorticity measurements in a single stream shear layer and its parent boundary layer. Diplomarbeit, Technische Hochshule, Aachen.

Bruns, J. M., Haw, R. C. \& Foss, J. F. 1991 The velocity and transverse vorticity field in a single stream shear layer. In Eighth Symp. on Turbulent Shear Flows, Technical University of Munich, September 9-11.

BruUn, H. H. 1995 Hot-wire Anemometry. Oxford University Press.

Dimotakis, P. E. 1986 Two-dimensional shear-layer entrainment. AIAA J. 24, 1791-1796.

Dimotakis, P. \& Brown, G. 1976 The mixing layer at high Reynolds number: large-structure dynamics and entrainment. J. Fluid Mech. 78, 535-560.

Dziomba, B. \& Fiedler, H. E. 1985 Effect of initial conditions on two-dimensional free shear layers. J. Fluid Mech. 152, 419-442.

Foss, J. F. 1977 The effect of the laminar/turbulent boundary layer states on the development of a plane mixing layer. Proc. 1st Intl Symp. on Turbulent Shear Flows, Penn. State Univ., p. 1133.

Foss, J. F. 1994 Vorticity considerations and planar shear layers. Expl Therm. Fluid Sci. 8, 260-270.

Foss, J. F. \& Haw, R. C. 1990 Transverse vorticity measurements using a companct array of four sensors. In The Heuristics of Thermal Anemometry (ed. D. E. Stock, S. A. Sherif \& A. J. Smits). ASME-FED vol. 97, pp. 71-76.

Hamelin, J. \& Alving, A. E. 1996 A low-shear turbulent boundary layer. Phys. Fluids 8, 789-804.

Ho, C. \& Huerre, P. 1984 Perturbed free shear layers. Annu. Rev. Fluid Mech. 16, 365-424.

Holmes, P., Lumley, J. L. \& Berkooz, G. 1992 Coherent Structures, Symmetry. Dynamical Systems and Turbulence. Cambridge University Press.

Hussain, A. K. M. F. \& Zaman, K. B. M. Q. 1985 An experimental study of organized motions in the turbulent plane mixing layer. J. Fluid Mech. 159, 85-104 (referred to herein as H-Z).

Hussain, A. K. M. F. \& ZeDan, M. F. 1978 Effects of the initial condition on the axisymmetric free shear layer: effects of the initial momentum thickness. Phys. Fluids 21, 1100-1112.

KLEWICKI, J. C. 1989 On the interactions between the inner and outer region motions in turbulent boundary layers. PhD thesis, Michigan State University, East Lansing, MI.

KLEWICKI, J. C. \& FALCO, R. E. 1990 On accurately measuring statistics associated with small-scale structure in turbulent boundary layers using hot-wire probes. J. Fluid Mech. 219, 119-142.

Klewicki, J. C., Falco, R. E. \& Foss, J. F. 1992 Some characteristics of the vortical motions in the outer region of turbulent boundary layers. Trans. ASME: J. Fluids Engng 114, 530-536.

Koochesfahani, M. M., Catherasoo, C. J., Dimotakis, P. E., Gharib, M. \& Lang, D. B. Two-point LDV measurements in a plane mixing layer. AIAA, J. 17, 1347-1351.

Marusic, I. \& Perry, A. E. 1995 A wall-wake model for the turbulence structyre of boundary layers. Part 2. Further experimental support. J. Fluid Mech. 298, 389-407.

Morris, S. C. 2002 The velocity and vorticity fields of a single stream shear layer. $\mathrm{PhD}$ thesis, Michigan State University, East Lansing, MI.

NAGUIB, A. 1992 Inner and outer layer effects on the dynamics of a turbulent boundary layer. $\mathrm{PhD}$ thesis, Illinois Institute of Technology, Chicago, IL. 
Narayanan, S. \& Hussain, F. 1996 Measurements of spatiotemporal dynamics in a forced plane mixing layer. J. Fluid Mech. 320, 71-115.

Panton, R. L. 2001 Overview of the self-sustaining mechanicsm of wall turbulence. Prog. Aerospace Sci. 37, 341-383.

Pope, S. 2000 Turbulent Flows. Cambridge University Press.

Saddoughi, S. G. \& Veeravalli, S. V. 1994 Local isotropy in turbulent boundary layers at high Reynolds number. J. Fluid Mech. 189, 333-372.

Slessor, M. D., Bond, C. L. \& Dimotakis, P. E. 1998 Turbulent shear-layer mixing at high Reynolds numbers: effects of inflow conditions. J. Fluid Mech. 376, 115-138.

Thomas, F. O. 1991 Structure of mixing layers and jets. Appl. Mech. Rev. 44, 119-153.

Townsend, A. A. 1975 The Structure of Turbulent Shear Flow. Cambridge University Press.

Wallace, J. M. \& Foss, J. F. 1995 The measurement of vorticity in turbulent flows. Annu. Rev. Fluid Mech. 27, 469-514. 